\title{
From cytogenetics to cytogenomics: whole- genome sequencing as a first-line test comprehensively captures the diverse spectrum of disease-causing genetic variation underlying intellectual disability
}

Anna Lindstrand ${ }^{1,2,3^{*}}$ (D), Jesper Eisfeldt ${ }^{1,2,3,4}$, Maria Pettersson ${ }^{1,2,3}$, Claudia M. B. Carvalho ${ }^{5}$, Malin Kvarnung ${ }^{1,2,3}$, Giedre Grigelioniene ${ }^{1,2,3}$, Britt-Marie Anderlid ${ }^{1,2,3}$, Olof Bjerin ${ }^{6}$, Peter Gustavsson 1,2,3 , Anna Hammarsjö 1,2,3, Patrik Georgii-Hemming ${ }^{1}$, Erik Iwarsson ${ }^{1,2,3}$, Maria Johansson-Soller ${ }^{1,2,3}$, Kristina Lagerstedt-Robinson 1,2,3, Agne Lieden 1,2,3, Måns Magnusson ${ }^{2,4,7}$, Marcel Martin ${ }^{8}$, Helena Malmgren ${ }^{1,2,3}$, Magnus Nordenskjöld ${ }^{1,2,3}$, Ameli Norling ${ }^{6}$, Ellika Sahlin ${ }^{1,2,3}$, Henrik Stranneheim ${ }^{2,3,7}$, Emma Tham $^{1,2,3}$, Josephine Wincent ${ }^{1,2,3}$, Sofia Ygberg ${ }^{6,7}$, Anna Wedell ${ }^{2,7}$, Valtteri Wirta ${ }^{9,10}$, Ann Nordgren ${ }^{1,2,3}$, Johanna Lundin ${ }^{1,3,5+}$ and Daniel Nilsson ${ }^{1,2,3,4 \dagger}$

\footnotetext{
Abstract

Background: Since different types of genetic variants, from single nucleotide variants (SNVs) to large chromosomal rearrangements, underlie intellectual disability, we evaluated the use of whole-genome sequencing (WGS) rather than chromosomal microarray analysis (CMA) as a first-line genetic diagnostic test.

Methods: We analyzed three cohorts with short-read WGS: (i) a retrospective cohort with validated copy number variants (CNVs) (cohort 1, $n=68$ ), (ii) individuals referred for monogenic multi-gene panels (cohort 2, $n=156$ ), and (iii) 100 prospective, consecutive cases referred to our center for CMA (cohort 3). Bioinformatic tools developed include FindSV, SVDB, Rhocall, Rhoviz, and vcf2cytosure.

\footnotetext{
* Correspondence: anna.lindstrand@ki.se

†'Johanna Lundin and Daniel Nilsson contributed equally to this work.

'Department of Clinical Genetics, Karolinska University Hospital, Stockholm,

Sweden

${ }^{2}$ Department of Molecular Medicine and Surgery, Karolinska Institutet,

Stockholm, Sweden

Full list of author information is available at the end of the article
}

(c) The Author(s). 2019 Open Access This article is distributed under the terms of the Creative Commons Attribution 4.0 International License (http://creativecommons.org/licenses/by/4.0/), which permits unrestricted use, distribution, and reproduction in any medium, provided you give appropriate credit to the original author(s) and the source, provide a link to the Creative Commons license, and indicate if changes were made. The Creative Commons Public Domain Dedication waiver (http://creativecommons.org/publicdomain/zero/1.0/) applies to the data made available in this article, unless otherwise stated. 
(Continued from previous page)

Results: First, we validated our structural variant (SV)-calling pipeline on cohort 1, consisting of three trisomies and 79 deletions and duplications with a median size of $850 \mathrm{~kb}$ (min $500 \mathrm{bp}$, max $155 \mathrm{Mb}$ ). All variants were detected. Second, we utilized the same pipeline in cohort 2 and analyzed with monogenic WGS panels, increasing the diagnostic yield to 8\%. Next, cohort 3 was analyzed by both CMA and WGS. The WGS data was processed for large (> $10 \mathrm{~kb}$ ) SVs genome-wide and for exonic SVs and SNVs in a panel of 887 genes linked to intellectual disability as well as genes matched to patient-specific Human Phenotype Ontology (HPO) phenotypes. This yielded a total of 25 pathogenic variants (SNVs or SVs), of which 12 were detected by CMA as well. We also applied short tandem repeat (STR) expansion detection and discovered one pathologic expansion in ATXN7. Finally, a case of Prader-Willi syndrome with uniparental disomy (UPD) was validated in the WGS data.

Important positional information was obtained in all cohorts. Remarkably, 7\% of the analyzed cases harbored complex structural variants, as exemplified by a ring chromosome and two duplications found to be an insertional translocation and part of a cryptic unbalanced translocation, respectively.

Conclusion: The overall diagnostic rate of $27 \%$ was more than doubled compared to clinical microarray (12\%). Using WGS, we detected a wide range of SVs with high accuracy. Since the WGS data also allowed for analysis of SNVS, UPD, and STRs, it represents a powerful comprehensive genetic test in a clinical diagnostic laboratory setting.

Keywords: Whole-genome sequencing, Intellectual disability, Monogenic disease, Copy number variation, Structural variation, Single nucleotide variant, Uniparental disomy, Repeat expansion

\section{Background}

Up to $80 \%$ of rare diseases have a genetic background [1], and in the last decade, our understanding of the underlying genetic lesions has increased rapidly. Since human genetic variation is abundant and diverse, ranging from small variants affecting only one or a few base pairs, i.e., single nucleotide variants (SNVs), small insertions and deletions (INDELs) to large structural variants (SVs) affecting thousands or millions of nucleotides [2-4], diagnostic tests need to capture the full spectrum of variation. Technological advancements in high-throughput sequencing (massively parallel sequencing, MPS) has allowed for comprehensive sequencing of many individuals from various populations [5-7] highlighting the vast complexity and abundance of rare and common genetic variation $[8,9]$.

In genetic diagnostics, the current toolbox includes a great variety of cytogenetic and molecular methodologies. Chromosomal microarray analysis (CMA), either comparative genomic hybridization (CGH) or SNP arrays, has long been the first-tier test used to identify copy number variants $(\mathrm{CNVs})$ in individuals with intellectual disability and neurodevelopmental disorders [10-12]. In recent years, MPS-based assays have been increasingly used in rare disease diagnostics, because of their high throughput and cost effectiveness in screening multi-gene panels for hereditary disorders [13]. Whole-exome sequencing (WES) is more and more widely used in clinical labs as a first-tier test, allowing detection of SNVs, INDELs, and CNVs covering multiple exons (typically > 2) [14-16]. In a recent meta-analysis, the diagnostic yield of WES in 3350 individuals with neurodevelopmental disorders was 36\%, ranging from 8 to $90 \%$, with the higher yield obtained after trio analysis of the affected individuals and both parents [17]. Even though isolated WES or WES in combination with CMA enables effective detection of both SNVs and CNVs [18], some types of variants such as balanced chromosomal rearrangements, small CNVs ( $<2$ exons), and trinucleotide repeat expansion disorders will be missed. Depending on the specific clinical presentation and the preferences of the referring physician and the laboratory performing the test, other assays such as Gbanded karyotyping, fluorescence in situ hybridization (FISH), FMR1 CGG repeat expansion analysis, PCR-based single gene analysis, and whole-genome sequencing (WGS) may then be performed [19].

Each individual method has intrinsic specific limitations which may result in causal variants being missed (e.g., mosaicism in probands) or misinterpreted (e.g., gene copy number gains consistent with triplications or higher order gains can be challenging to distinguish from duplications [20]), resulting in sub-optimal clinical management and imprecise genetic counseling [21]. In addition, the possibility of dual diagnosis due to multilocus variation [22] has been reported for up to $5 \%$ of individuals with Mendelian diseases and can explain apparent phenotypic expansion [23]. In research, WGS has been used to detect a wide range of mutations, including copy number variations [24-26] as well as balanced chromosomal rearrangements such as translocations [27, 28], inversions [29], and short tandem repeats (STRs) [30]. A few studies have performed CNV calling from WGS in small cohorts, showing diagnostic rates of $15 \%$ (10/79) [24], 33\% (20/60) [31], and 14\% (7/50) [32]. Although WGS is the most comprehensive test currently available for molecular diagnostics in clinical practice, 
the routine use of WGS largely remains limited to SNVs and INDELs [33, 34]. This is because WGS-based SV detection in a clinical setting remains challenging, partly because of the low precision and sensitivity of the SV callers and lack of normal variant databases, but also due to the limited standardization and benchmarking of the various pipelines [35].

In this study, we investigate the application of WGS as a first-line test in intellectual disability and compare the outcome with results from CMA. In aggregate, the results highlight the ability to capture a broad range of genetic variation including both large and small CNVs, SNVs, balanced rearrangements, repeat expansions, and uniparental disomy (UPD). In a prospective unselected cohort of 100 patients referred to our laboratory for CMA, the overall diagnostic yield of WGS was $27 \%$ compared to $12 \%$ obtained with our standard clinical CMA.

\section{Methods}

\section{Study subjects}

Clinical Genetics (Karolinska University Hospital, Stockholm, Sweden) is a tertiary center where genomewide screening for CNVs by CMA is used as a first-line test for individuals with suspected rare genetic disease, neurodevelopmental disorders (NDD), and malformation syndromes. For individuals with a high suspicion of a monogenic disease, WGS (with in silico gene panel analysis) is performed as the first-line test. Overall, roughly 1000 CMAs and 500 WGS analyses are performed annually. In this study, all included patients were initially referred for clinical diagnostic testing and, when possible, parental analysis was performed to assess the parental origin of identified variants. Three cohorts were investigated:
Cohort 1, "The validation cohort", consisted of 68 individuals harboring three trisomies and $79 \mathrm{CNVs}$ previously detected by CMA or multiplex ligationdependent probe amplification (MLPA).

Cohort 2, "The monogenic disease study cohort", consisted of 156 individuals referred for WGS due to a clinical suspicion of monogenic disease within the areas of neuromuscular disorders, connective tissue disorders, unknown syndromes, skeletal dysplasias, hereditary cancer, or other rare suspected Mendelian conditions.

Cohort 3, "The prospective study cohort", consisted of the first 100 unselected individuals that were clinically referred for CMA in 2017.

The clinical parameters of the three cohorts are summarized in Table 1 and detailed information is given in Additional file 1: Table S1. The local ethical board in Stockholm, Sweden, approved the study (approval numbers KS 2012/222-31/3 and 2012/2106-31/4).

\section{Chromosomal microarray analysis}

Genomic DNA was isolated from whole blood using standardized protocols and used for array comparative genomic hybridization (array-CGH) analysis. A $4 \times 180 \mathrm{~K}$ custom oligonucleotide microarray with whole-genome coverage and a median probe spacing of approximately $18 \mathrm{~kb}$ was used (AMADID:031035, Oxford Gene Technology, Begbroke, Oxfordshire, UK). This array design is used as a routine diagnostic tool at the Department of Clinical Genetics, Karolinska University Hospital, Stockholm, Sweden. For sample RD_P409, a medical exome $1 \times 1 \mathrm{M}$ Agilent oligonucleotide microarray was used (AMADID: 068073, Oxford Gene Technology, Begbroke, Oxfordshire,

Table 1 Clinical parameters of included cases

\begin{tabular}{|c|c|c|c|}
\hline Clinical & Cohort $1(n=68)$ & Cohort $2(n=156)$ & Cohort $3(n=100)$ \\
\hline Gender (F/M) & $44 \% / 56 \%$ & $44 \% / 50 \% *$ & $37 \% / 63 \%$ \\
\hline \multicolumn{4}{|l|}{ Main phenotype } \\
\hline NDD & $21(31 \%)$ & $2(1 \%)$ & $40(40 \%)$ \\
\hline NDD+ & 13 (19\%) & 0 & $38(38 \%)$ \\
\hline Syndrome & $20(29 \%)$ & $53(34 \%)$ & $8(8 \%)$ \\
\hline Growth abnormality & $5(7 \%)$ & 0 & $4(4 \%)$ \\
\hline Metabolic crisis & 0 & 0 & $3(3 \%)$ \\
\hline Endocrine abnormality & $2(3 \%)$ & 0 & $1(1 \%)$ \\
\hline Internal malformations & $1(1 \%)$ & 0 & $2(2 \%)$ \\
\hline Neuromuscular abnormality & $1(1 \%)$ & $32(21 \%)$ & $2(2 \%)$ \\
\hline CTD & 0 & $28(18 \%)$ & 0 \\
\hline Hereditary cancer & $3(4 \%)$ & $33(21 \%)$ & 0 \\
\hline Other** & $2(3 \%)$ & $8(5 \%)$ & $2(2 \%)$ \\
\hline
\end{tabular}

$F$ female, $M$ male, NDD neurodevelopmental disorder, NDD+ syndromic NDD, CTD connective tissue disorder. ${ }^{*} 6 \%$ no gender information (fetal sample or disorder of sex development). **Epilepsy, disorder of sex development, eye disorder, immunological disorder, and skin disease 
UK). The medical exome $1 \times 1 \mathrm{M}$ array is an exonfocused array used for targeted analysis of deletions/ duplications falling below the resolution of the $4 \times$ $180 \mathrm{~K}$ standard microarray. The probes have been selected to allow for single exon resolution in 4645 known disease-causing genes.

The control DNA used for the array-CGH experiment consisted of a mix of sex-matched DNA from several healthy individuals pooled together (Promega, Madison, WI, USA). Sample labelling (CGH labelling kit for oligo arrays, Enzo Life Sciences, Farmingdale, NY, USA), hybridization, and slide washing (Oligo aCGH/ChIP-onChip Wash Buffer Kit, Agilent Technologies, Wilmington, DE, USA) were performed according to the manufacturers' recommendations. Slides were scanned using the Agilent Microarray Scanner (G2505C, Agilent technologies, USA) with $3 \mu \mathrm{m}$ resolution. Raw data were normalized using Feature Extraction Software v10.7.3.1 (Agilent Technologies, Santa Clara, CA, USA), and $\log 2$ ratios were calculated by dividing the normalized intensity in the sample by the mean intensity across the reference sample. The $\log 2$ ratios were plotted and segmented by circular binary segmentation in the CytoSure Interpret software v4.10 (Oxford Gene Technology, Oxfordshire, UK). Oligonucleotide probe positions were annotated according to the human genome assembly hg19 [36]. For the $4 \times 180 \mathrm{~K}$ microarray, three consecutive aberrant probes with a $\log 2$ ratio cutoff of -0.65 for deletions and 0.35 for duplications were called, giving a practical lower resolution of about $50 \mathrm{~kb}$. The clinical relevance of all CNVs was classified into five categories; benign, likely benign, variant of uncertain significance (VUS), likely pathogenic, and pathogenic, according to the American College of Medical Genetics and Genomics (ACMG) guidelines [37] and based upon the size of aberration, gene content, inheritance, and available information in medical literature and different databases: the Database of Genomic Variants (DGV) [38], the Database of Chromosomal Imbalance and Phenotype in Humans using Ensembl Resources (DECIPHER) [39], Online Mendelian Inheritance in Man (OMIM) [40], and an in-house database with variants from $\sim 8000$ analyzed cases.

\section{Multiplex ligation-dependent probe amplification}

Multiplex ligation-dependent probe amplification (MLPA) analysis was performed using the available probe set for selected assays (P090 (BRCA2), P256 (FLCN), P003 (MLH1), and ME028 (PWS) MRC-Holland, Amsterdam, Netherlands). MLPA was carried out according to the supplier's recommendations, with the exception that the PCR reactions were performed in a $25-\mu \mathrm{l}$ reaction volume. Amplification products were quantified by capillary electrophoresis on an ABI3500xL Genetic Analyzer (Applied Biosystems, Thermo Fisher Scientific, Waltham, MA,
USA) with accompanying software. The tracing data was imported into and analyzed in GeneMarker software v1.7 (SoftGenetics LLC, State College, PA, USA). The normalized quotients for the different probes were considered as a deletion when below 0.75 and a duplication when above 1.3.

\section{Genotyping}

Genomic DNA from individual RD_P432 and her mother were analyzed using 12 polymorphic microsatellite markers located on chromosome 15 (D15S1035, D15S128， D15S1513， D15S97， D15S1002， D15S165, D15S1007, D15S123, D15S1024, D15S992, D15S1028, and D15S978). Primers were pooled and amplified using Type-it Microsatellite PCR Kit according to the manufacturer's instructions (QIAGEN, Hilden, Germany). PCR products were analyzed using 3500xL Genetic Analyzer and GeneMapper v5 according to the manufacturer's protocol (Applied Biosystems).

\section{Short-read whole-genome sequencing}

Genomic DNA from whole blood was sequenced using the Illumina Hiseq X Ten platform, using a 30× PCR-free paired-end WGS protocol. The patients of cohort $1(n=$ 68) were sequenced at the National Genomics Infrastructure (NGI), Stockholm, Sweden [41], and the patients of cohort $2(n=156)$ and cohort $3(n=100)$ were sequenced at Clinical Genomics, Stockholm, Sweden [42]. The resulting WGS data was preprocessed according to the GATK best practices for germline WGS data [43]. SVs were analyzed using the FindSV pipeline [44], a pipeline combining CNVnator V0.3.2 [45] and TIDDIT V2.0.0 [46]. The outputs of these callers ( 27,000 SVs; Additional file 2: Figure S1) are merged using SVDB [47], and the resulting variant calling file (VCF) is annotated using variant effect predictor (VEP) 87 [48]. Finally, the VCF is annotated and sorted based on the allele frequencies in the Swedish structural variant frequency database (SweFreq SVDB) $[6,49]$, as well as an internal database (internal SVDB) consisting of approximately 400 individuals.

SNVs were called using MIP [34], a pipeline that combines Samtools [50], FreeBayes [51], and the GATK HaplotypeCaller [43] generating an average of 5,500,000 SNVs and INDELS (Additional file 2: Figure S2). Finally, ExpansionHunter v2.5.5 [30] was applied to the Illumina short-read whole-genome alignment files produced by MIP v.6.0.0, assessing STRs in 17 genes (AR, ATN1, ATXN1, ATXN10, ATXN2, ATXN3, ATXN7, C9ORF72, CACNA1A, CBL, CSTB, DMPK, FMR1, FXN, HTT, $J P H 3$, and $P P P 2 R 2 B)$.

\section{Data analysis of WGS data in the prospective study}

The WGS data was analyzed in three steps: (1) large $\mathrm{CNVs}$, (2) small $\mathrm{CNVs}$ and genomic rearrangements, 
and (3) SNVs, INDELS, and repeat expansions. A panel of genes linked to intellectual disability (ID gene panel) that consisted of 887 genes was generated based on the information available at the time through the Genomics England panel app [52] (Additional file 2: Document S1) which was used for filtering small CNVs, SNVs, and INDELS. In addition, for individuals with other clinical symptoms, personalized gene panels were generated based on the patient-specific Human Phenotype Ontology (HPO) $[53,54]$ terms using the database available through Charité [55] to link the HPO terms and genes.

For SV analysis (aforementioned steps 1 and 2), the output data from FindSV was filtered based on variant size (intergenic variants $>10 \mathrm{~kb}$, and intragenic variants $>2 \mathrm{~kb}$ ), quality (minimum 6 read pairs (TIDDIT), minimum $5 \mathrm{~kb}$ in size (CNVnator)), and allele frequency (SweFreq SVDB $<0.5 \%$, internal SVDB $<1 \%$ ), and calls located on the decoy contig were also filtered out. Finally, a list of SVs with junctions located within the ID or HPO gene list was generated without a size cutoff. The number of SVs remaining after each filtering step is available in the supplemental data (Additional file 2: Figure S1).

Step 1: To visualize large CNVs, we used vcf2cytosure [56] that converts a VCF with structural variations to the ".CGH" format used by the CytoSure Interpret Software by OGT. CytoSure is normally used in our laboratory to interpret the clinical significance of CNVs detected by CMA. By displaying the WGS data in a familiar system where we have access to a large internal database of previously assessed cases, variant

classification is facilitated (Additional file 2: Figure S3). As described in the CMA section, detected CNVs were classified into five categories according to the ACMG guidelines [57].

Step 2: Selected SVs were visualized in a list view enabling more detailed assessment of balanced/complex genomic rearrangements. Indications of a genomic rearrangement include (i) several SVs in the same genomic region, (ii) discordant read pairs mapping from a duplication/deletion boundary to another chromosomal region, and (iii) several CNVs clustering on the same chromosome. All such variants of interest were then inspected in IGV, and the derivative chromosomes were reconstructed by visual inspection and breakpoint junction analysis [58].

Step 3: SNVs and INDELS were annotated using MIP [34] and repeat expansions with ExpansionHunter [30]. Rare variants with a minor allele frequency (MAF) less than 1\% in Exome Aggregation Consortium (ExAC) $[8,59]$ or in the Swedish variant frequency database (SweFreq) $[6,60]$ and located within the ID or HPO gene list were considered for further analysis. We then used an internal scoring system that also takes into account conservation and predicted severity of the variant [34] that is available on GitHub [61]. The number of SNVs remaining after each filtering step is available in the supplemental data (Additional file 2: Figure S2). Finally, remaining variants were individually assessed and classified according to ACMG guidelines [57] into five classes; benign, likely benign, likely pathogenic, pathogenic, and uncertain significance.

All SNVs and CNVs reported in this study have been submitted to the ClinVar database [62].

Finally, in individual RD_P432, maternal uniparental disomy (UPD) for chromosome 15 was assessed in the WGS data. In brief, this was done in two steps using inhouse developed bioinformatic tools. First, we searched for regions of homozygosity on chromosome 15 using rhocall [63] and then we made a run of homozygozity (RoH) plot using the rhoviz tool from the same package. In brief, this assesses zygosity ratios at all SNPs across an entire chromosome (in this case chromosome 15). The zygosity ratio is defined as the ratio of high-quality variants in a local region supporting a heterozygous allele to the total number of high-quality variants in that region.

\section{Verification of variants identified by WGS}

SNVs were verified with PCR and Sanger sequencing. For the two deletions in MBD5 and C12orf65 identified by WGS in individuals RD_P416 and RD_P417, respectively, and the reciprocal translocation between chromosomes 4 and 7 (RD_P77) (Table 3), primers flanking the breakpoints were designed approximately 500 base pairs away from the estimated breakpoints. The same primers were subsequently used for sequencing using the Sanger method (primer sequences available upon request). The PCR was performed using standard methods with Platinum Taq DNA Polymerase (Invitrogen, Carlsbad, CA, USA). Sequences were aligned using BLAT (UCSC Genome Browser) $[64,65]$ and visualized in the CodonCode Aligner software (CodonCode Corp., Dedham, MA, USA).

The ATXN7 STR identified in individual RD_P431 was verified and the exact number of CAG copies determined using PCR followed by fragment length analysis [66]. The length of the PCR products was determined using capillary electrophoresis on an ABI3500xL Genetic Analyzer and the software Gene Mapper v5 (Applied Biosystems, Thermo Fisher Scientific, Waltham, MA, USA).

Fluorescence in situ hybridization (FISH) was performed using standardized protocols from peripheral blood cultures from patient RD_P405, harboring a duplication on chromosome 3 where the WGS data suggested that the duplication had been inserted on chromosome 13. Probes used were RP11-209H21-SG (green, chr3:159, 
243,721-159,420,409 (Hg19)) and RP11-203L15SO (red, chr3:160,561,956-160,724,921 (Hg19)).

Chromosome analysis was performed on metaphases from peripheral blood cultures from patients RD_P406 and RD_P414 according to standard protocols with subsequent G-banding with an approximate resolution of 550 bands per haploid genome. A total of 12 metaphases were analyzed.

\section{Results}

WGS reliably identifies deletions, duplications, and aneuploidies and reveals additional clinically relevant genetic information

To validate the SV calling pipeline, a total of 68 individuals with three trisomies and $79 \mathrm{CNVs}$, previously detected by CMA (65 individuals) or MLPA (three individuals), including 54 deletions and 25 duplications, were subjected to short-read WGS sequencing (Fig. 1, Table 2, Additional file 1: Table S1) and analyzed with the FindSV pipeline that includes the two SV callers: CNVnator [45] and TIDDIT [46]. All validation CNVs were detected in the WGS data. The two callers performed slightly differently, mainly depending on whether the $\mathrm{CNV}$ breakpoints were located in repetitive regions (Table 2). The size distribution of the variants ranged from $500 \mathrm{bp}$ (single exon $\mathrm{CNVs}$ ) to $155 \mathrm{Mb}$ (whole chromosome) (Fig. 1, Additional file 1: Table S1).

\section{Deletions}

A total of 54 deletions were identified in cohort 1 , including seven recurrent (13\%) and 47 non-recurrent (87\%) (Table 2). The size ranged between $5 \mathrm{~kb}$ and $47 \mathrm{Mb}$ (Fig. 1, Additional file 1: Table S1). TIDDIT identified a total of 39 deletions (83\%), all of which were non-recurrent while CNVnator identified a total of 51 deletions (94\%), including all recurrent deletions (Table 2). The seven recurrent deletions included four contiguous gene syndromes: DiGeorge syndrome (22q11.21 deletion; MIM 188400), Chromosome 1p36 deletion syndrome (MIM 607872), Chromosome 16p11.2 deletion syndrome (MIM 611913), and Prader-Willi syndrome (MIM 176270) due to a 15q11.2 deletion (Additional file 1: Table S1).

\section{Duplications}

A total of 23 unique duplications were identified in cohort 1 , five of which were recurrent (22\%) and 18 were
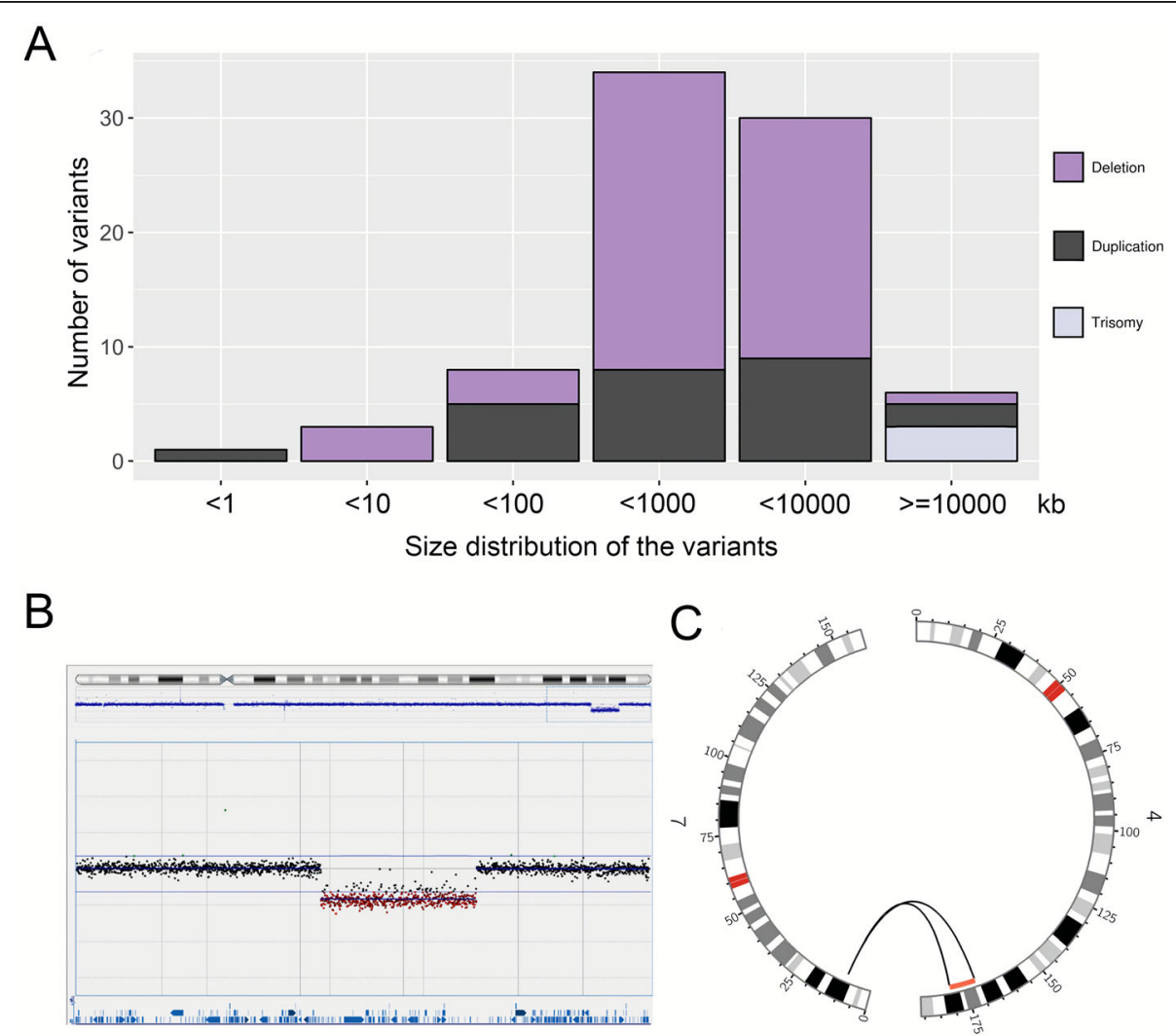

Fig. 1 Overview of CNVs and affected individuals included in the validation cohort. a Bar graph showing the size distribution of 79 validated CNVs and three trisomies that were detected with WGS. Deletions are shown in purple, duplications in black, and trisomies in lilac. b Array comparative genomic hybridization plot indicates a heterozygous deletion of $9.3 \mathrm{Mb}$ in individual RD_P77. c Circos plot illustrating the WGS results in the same individual. Discordant read pairs between chromosomes 4 and 7 are shown as gray lines, and the deletion is shown in red 
Table 2 Overview of the 80 validated CNVs detected by CMA and WGS in 68 patients

\begin{tabular}{llll}
\hline Type & Found by TIDDIT & Found by CNVnator & Found by CMA \\
\hline Deletions & & & $7(100 \%)$ \\
$\quad$ Recurrent [7] & $0(0 \%)$ & $44(94 \%)$ & $7(100 \%)$ \\
$\quad$ Non-recurrent [47] & $39(83 \%)$ & $51(94 \%)$ & $50(93 \%)$ \\
$\quad$ Total [54] & $39(72 \%)$ & $5(100 \%)$ & $5(100 \%)$ \\
Duplications & & $16(89 \%)$ & $15(83 \%)$ \\
$\quad$ Recurrent [5] & $0(0 \%)$ & $20(87 \%)$ & $21(91 \%)$ \\
$\quad$ Non-recurrent [18] & $15(83 \%)$ & & $3(100 \%)$ \\
$\quad 15(65 \%)$ & $3(100 \%)$ &
\end{tabular}

CMA chromosomal microarray, WGS whole-genome sequencing

non-recurrent (78\%) (Table 2). The size of the duplications ranged between $474 \mathrm{bp}$ and $36.4 \mathrm{Mb}$ (Fig. 1, Additional file 1: Table S1). All five recurrent duplications were detected by CNVnator. The recurrent duplications included two contiguous gene syndromes, the 22q11.21 duplication syndrome (MIM 608363; two individuals) and the 16p11.2 duplication syndrome (MIM 614671) (Additional file 1: Table S1). Two duplications were part of complex rearrangements and are discussed below. Of the 16 unique simple duplications, 13 (81\%) were found by TIDDIT, allowing for determination of the positioning of the duplicated segments. It was found that all those duplications were in tandem (Table 2, Additional file 1: Table S1).

\section{Trisomies}

Three of the individuals in cohort 1 harbored chromosomal aneuploidies, one case with a mosaic trisomy 9 (RD_P167), one with trisomy 21 (RD_P25), and one with trisomy X (RD_P101) (Additional file 1: Table S1). In all cases, the trisomy was detected by $\mathrm{CNVnator}$ and verified with the coverage track provided by TIDDIT [46, 67]. In individual RD_P167, the mosaicism level was estimated to be $\sim 46 \%$ with CMA and karyotyping showed it to be present in $\sim 29 \%(15 / 51)$ of analyzed metaphases. The WGS data confirmed the presence of a mosaic trisomy 9 present in $\sim 46 \%$ of cells.

\section{Complex rearrangements}

Three rearrangements in the validation cohort were known to be complex from the molecular cytogenetics analysis: RD_P22 (DEL-NML-DEL), RD_P07 (DEL-NMLDEL-NML-DUP), RD_P05 (DEL-DUP-DEL) (DEL; deletion, NML; normal, DUP; duplication) (Additional file 1: Table S1), and in an additional five individuals $(5 / 68,7 \%)$, the WGS analysis identified unexpected complexities compared to the original molecular cytogenetic results. These five unexpectedly complex cases included two
DEL-INV-DEL rearrangements (RD_P54, RD_P26) and two DUP-NML-DUP rearrangements (RD_P106, RD P105). The results from those eight cases have been reported previously in an article focused on the detailed characterization of clustered CNVs [68].

In addition, a $9.3-\mathrm{Mb}$ de novo deletion on chromosome 4 turned out to be part of a reciprocal translocation between chromosomes 4 and 7 (RD_P77) (Fig. 1, Additional file 1: Table S1). The reciprocal translocation was confirmed with breakpoint junction PCR that confirmed the presence of both junctions. The breakpoint junction analysis of the rearrangement $(\mathrm{t}(4 ; 7)(\mathrm{q} 33 ; \mathrm{p} 21.3))$ revealed non-templated insertions of 8 and 5 nucleotides in both junctions and no microhomologies, consistent with non-homologous end-joining (NHEJ), the most prevalent formation mechanism underlying reciprocal translocations [27, 69] (Additional file 2: Figure S4).

\section{Implementation of WGS-SV calling in monogenic rare diseases increases the diagnostic yield}

Next, we explored the diagnostic yield of gene-focused SV analysis in 156 patients referred for genetic investigation due to a clinical suspicion of monogenic disease. At the Karolinska University Hospital, we have since 2015 used WGS in individuals with a clinical suspicion of monogenic diseases to screen for SNVs and INDELs in multi-gene panels created by in silico filtering of the WGS data. Here we sought to implement SV calling focused on variants within the patient-specific in silico panel. In this way, we identified 12 clinically relevant SVs (8\%): eight deletions, two duplications, one inversion, and one complex rearrangement with two deletions and an inversion (DELINV-DEL) (Table 3, Fig. 2) increasing the diagnostic yield.

\section{A founder duplication in LAMA2 is the cause of neuromuscular disease in two unrelated individuals}

An identical homozygous duplication of exon 30 in LAMA2 was found in two seemingly unrelated individuals 


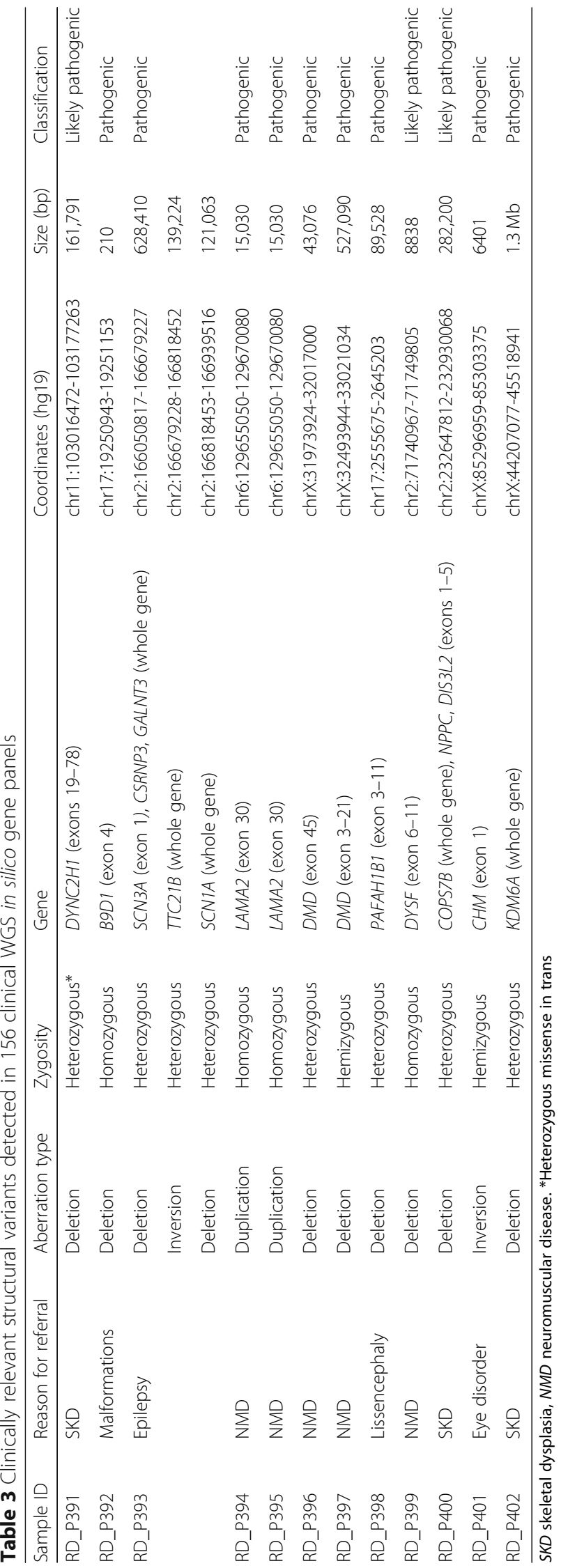



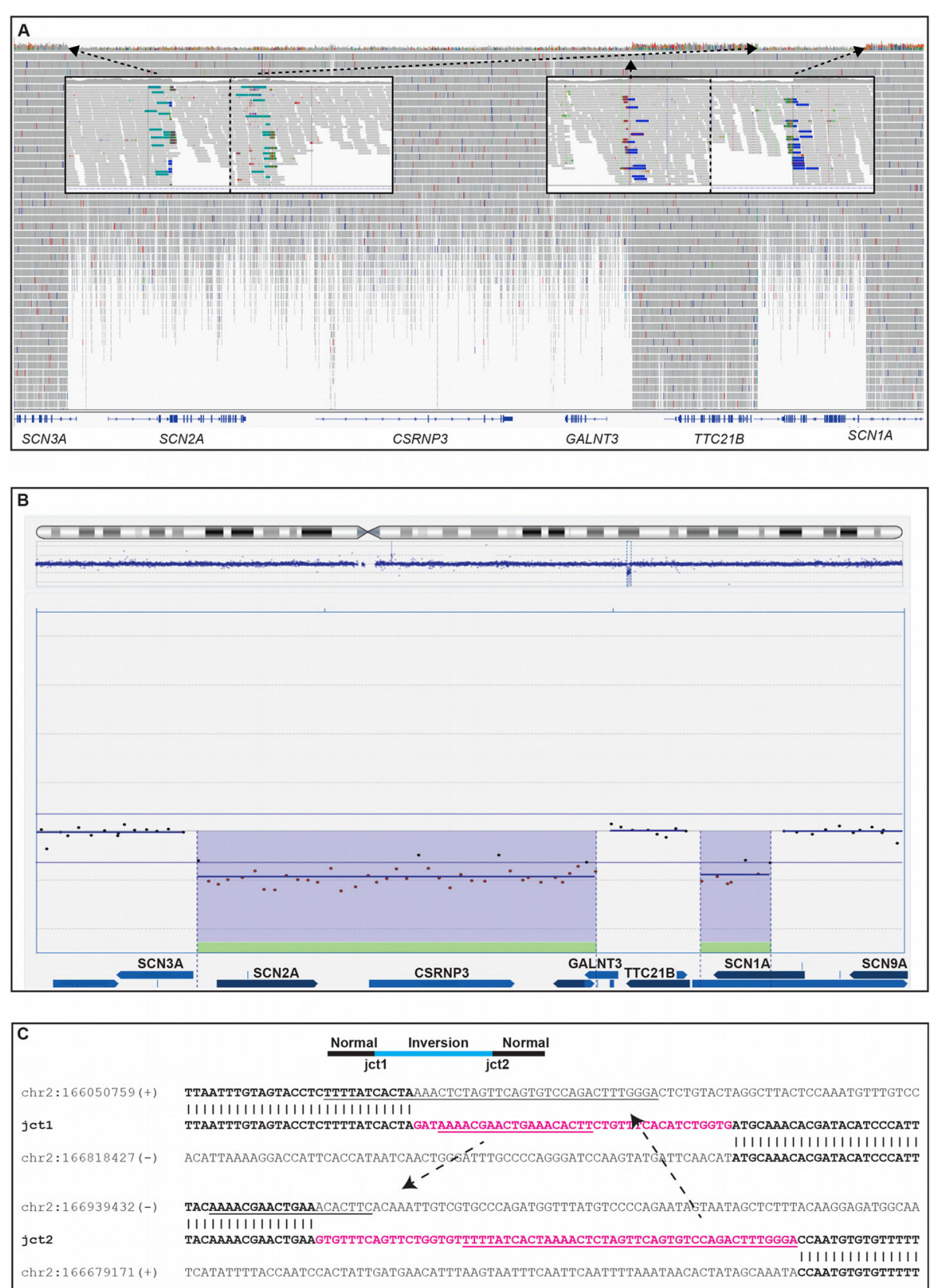

Fig. 2 A complex DEL-INV-DEL rearrangement identified by WGS causes severe epilepsy. a Screenshot of the deletions and inversion from the Integrative Genomics Viewer (IGV) in individual RD_P393. Short-read whole-genome sequencing (WGS) detected two clustered deletions of $630 \mathrm{~kb}$ (SCN3A, SCN2A, CSRNP3, GALNT3) and $121 \mathrm{~kb}$ (SCN1A), respectively. The genomic segment of normal copy number state in-between the deletions $(139 \mathrm{~kb}, \pi C 21 B)$ had been inverted. Both inversion breakpoint junctions are shown with the green and blue bars corresponding to discordant reads with mates located on the other side of the inversion. $\mathbf{b}$ Screenshot of DEL-INV-DEL rearrangement confirmed by array comparative genomic hybridization (array-CGH). Screenshot from the Cytosure Interpret Software. The deletions in the rearrangement were confirmed using array-CGH. c Breakpoint junction sequences. Sequence analysis of the breakpoint junctions revealed insertions in both junctions of $38 \mathrm{bp}$ and $59 \mathrm{bp}$, respectively (pink). Substantial parts of the insertions had been templated from sequences involved in the rearrangement (underlined), suggestive of a replicative error as the underlying mechanism of formation. L1 repetitive elements were present in two of the breakpoints but did not form any fusion L1 elements. Lowercase letters indicate deleted sequences

of the same ethnic origin (RD_P394, RD_P395; Table 3) with muscular dystrophy. The duplication was not found in DGV, The Genome Aggregation Database (gnomAD) [70], or the SweFreq SVDB databases. LAMA2 (located on chromosome 6q22-23) consists of 65 exons and encodes the protein merosin. The WGS data showed signals consistent with a tandem duplication, which conceptually will produce one normal copy of exon 30 followed by a 
frameshift and a stop codon after 35 aberrant residues. Immunohistochemical antibody staining of merosin in muscle biopsies from both patients showed absence of the protein, consistent with a loss-of-function mutation and conforming to a diagnosis of autosomal recessive muscular dystrophy (MIM 607855) [71].

\section{A heterozygous deletion on 2927 causes dominant skeletal dysplasia}

In individual RD_P400 (Table 3), CMA and WGS analysis identified a maternally inherited $282-\mathrm{kb}$ deletion on chromosome 2q27 affecting three genes, the entire COPS7B, NPPC, and exons $1-5$ of DIS3L2. The patient had disproportionate short stature, brachydactyly E, and small hands and feet. Her phenotype is summarized in Additional file 2: Document S2. Recently, two unrelated families were reported with heterozygous missense variants in NPPC segregating with a short stature and small hand phenotype, very similar to that of our patient and her mother, have been described [72] (Additional file 2: Document S2). Further, NPPC plays an important role in endochondral ossification through regulation of chondrocyte proliferation and differentiation in the cartilaginous growth plate [73]. It binds and stimulates the cGMP production of the NPR2 receptor and is through that pathway involved in the pathogenesis of relatively common skeletal dysplasias such as achondroplasia (MIM 100800) and acromesomelic dysplasia, Maroteaux type (MIM 602875).

\section{A complex intrachromosomal rearrangement disrupting three epilepsy genes}

A DEL-INV-DEL rearrangement was identified by WGS in individual RD_P393, a girl with developmental delay and severe epilepsy. A detailed clinical description is available in the supplemental information (Additional file 2: Document S2). Due to a clinical suspicion of monogenic disease, WGS was performed and filtered for genes involved in inborn errors of metabolism as well as epilepsy without identifying a causative genetic variant. The WGS data was then analyzed for structural variants using the FindSV pipeline, and the results showed a de novo complex rearrangement on chromosome 2q24.2 including two heterozygous deletions separated by an inverted fragment. The two deletions were visible by CMA and directly affected four genes (SCN1A, $S C N 2 A, S C N 3 A$, and CSRNP3) (Fig. 2a, b, Table 4). The proximal deletion was $628 \mathrm{~kb}$, and the proximal breakpoint disrupted intron 1 of $S C N 3 A$ and the distal breakpoint were located $28 \mathrm{~kb}$ upstream of GALNT3, with a complete heterozygous loss of GALNT3, SCN2A, and CSRNP3. The distal deletion was $121 \mathrm{~kb}$, with the proximal breakpoint $28 \mathrm{~kb}$ downstream of $S C N 1 A$ and the distal breakpoint in intron 1 of SCN1A. Finally, the 139 $\mathrm{kb}$ genomic segment located in-between the deletions had been inverted resulting in a DEL-INV-DEL pattern (Fig. 2). Analysis of the breakpoint junctions revealed partially templated insertions of $38 \mathrm{bp}$ and $59 \mathrm{bp}$, respectively (Fig. 2c). The insertions did not originate from the sequences in direct proximity of each junction, instead parts of the insertion in junction 1 originated from junction 2 sequences, and parts of the insertion in junction 2 originated from junction 1 sequences (Fig. 2c). One breakpoint disrupted $S C N 1 A$ in intron 1 and another breakpoint disrupted $S C N 3 A$ in intron 1, with no surrounding repetitive sequences. The remaining two breakpoints did not disrupt any protein coding genes but were located in repetitive regions belonging to the L1 family (L1MC4a and L1MA4). No L1 fusion elements were created as a result of the rearrangement.

To summarize, this individual is a carrier of a structural variant that leads to loss of function in three distinct Mendelian epilepsy genes. Both deletions and duplications, involving each of SCN1A, SCN2A, and $S C N 3 A$, have been presented previously in cases with severe epileptic encephalopathies and developmental delay $[74,75]$. In the case presented here, the deletions disrupted $S C N 1 A$ and $S C N 3 A$ and resulted in a complete loss of SCN2A (Fig. 2, Table 3, Table 4). The clinical presentation is coherent with Dravet syndrome (MIM 607208), usually caused by mutations involving SCN1A; however, the phenotypic variability in SCN1A mutation carriers is wide [76]. In addition, mutations in SCN2A also cause epileptic encephalopathy (MIM 613721) and missense variants in SCN3A have been implicated in focal epilepsy in children [77].

\section{Prospective study of comprehensive WGS analysis in 100 cases referred for CMA}

Finally, we performed a prospective pilot study where the first 100 cases referred to the Department of Clinical Genetics (Karolinska University Hospital, Stockholm, Sweden) for CMA in 2017 were analyzed in parallel with WGS. The obtained WGS data were processed for large SVs (>10 kb), for genome-wide and small SVs $(>2 \mathrm{~kb})$, and for SNVs and INDELs in 887 genes linked to intellectual disability (Additional file 2: Document S1). For individuals with additional clinical symptoms, custom in silico gene panels were created using HPO terms and data were processed for small SVs in the same way as for the ID gene panel. Next, small $(>2 \mathrm{~kb})$ intragenic SVs were assessed in the both gene panels. Finally, we applied the ExpansionHunter analysis pipeline [30] to identify large expansions of STRs in 17 genes.

\section{Detection of copy number variants}

The CMA analysis identified, in 14 patients, a total of 15 CNVs that were classified as pathogenic $(n=10)$, likely pathogenic $(n=3)$, and variants of uncertain significance 


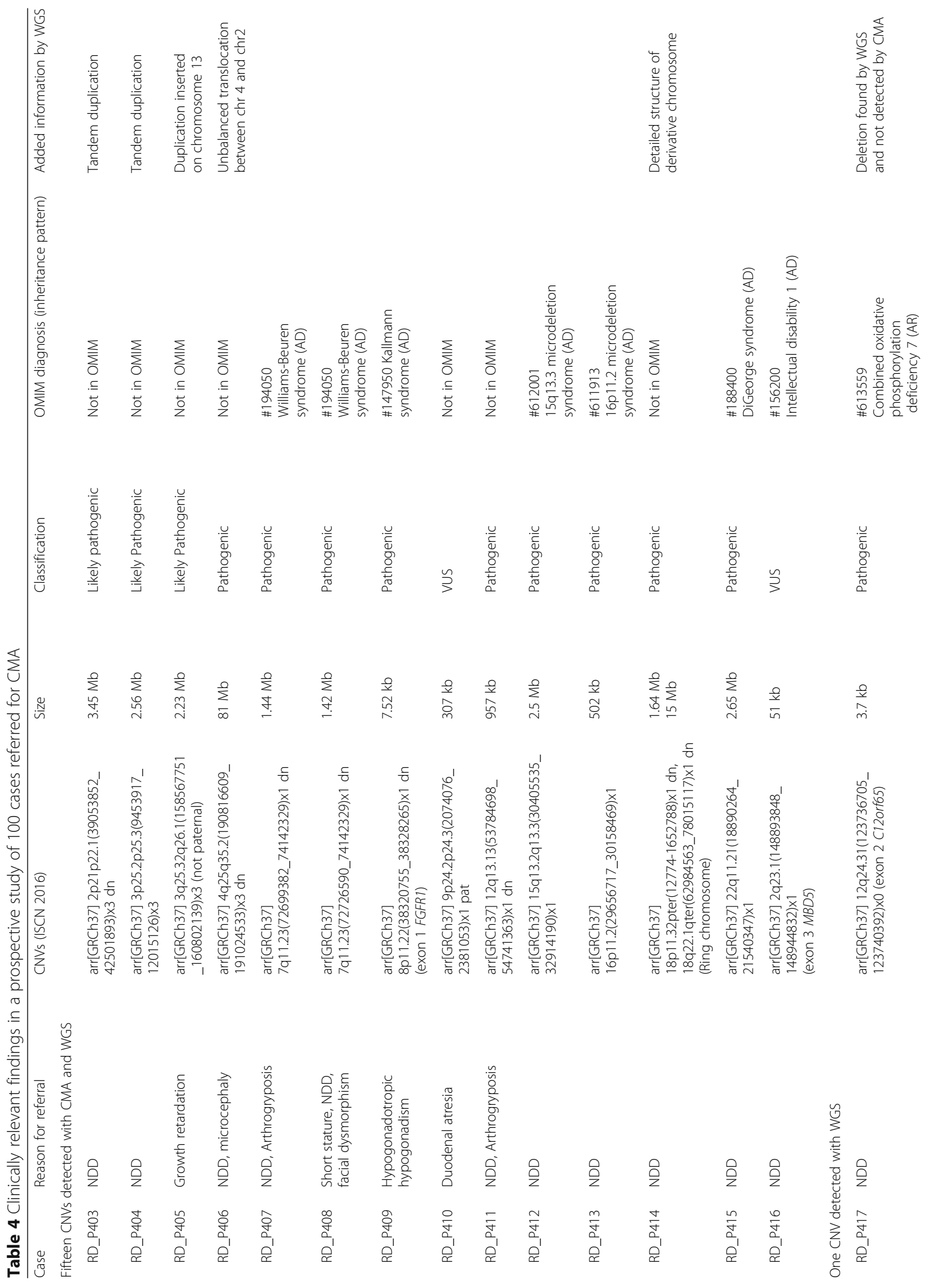




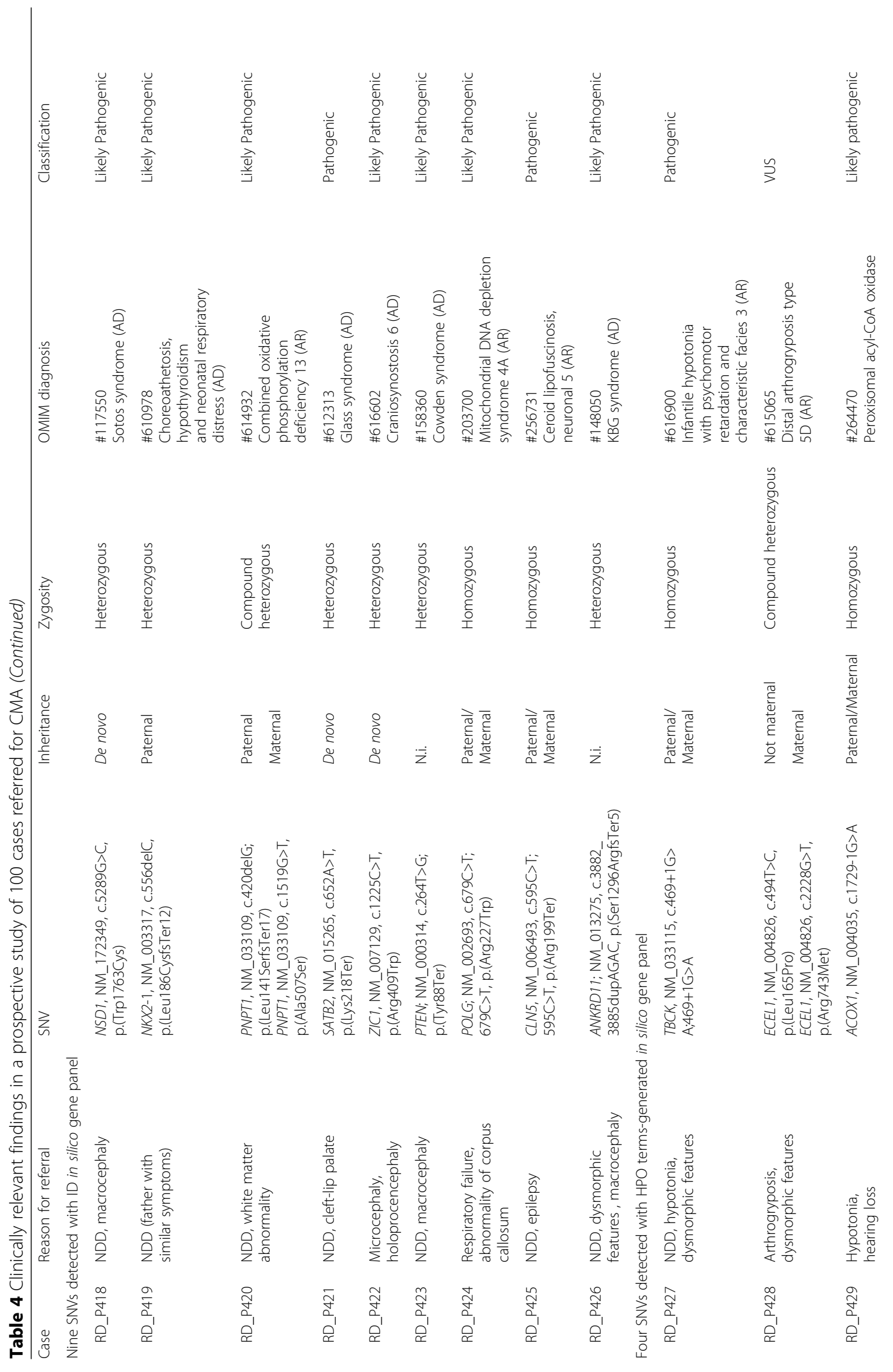




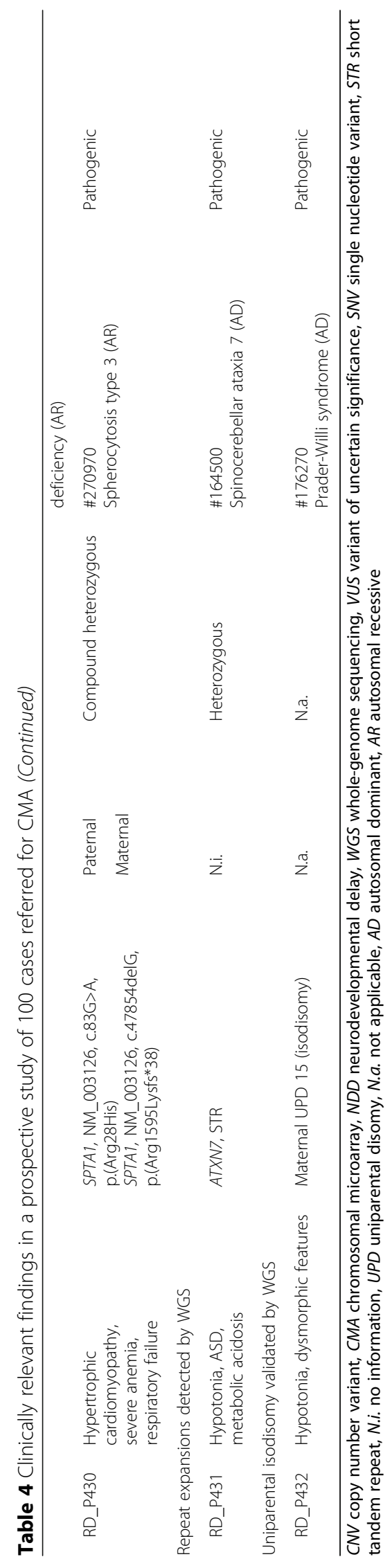



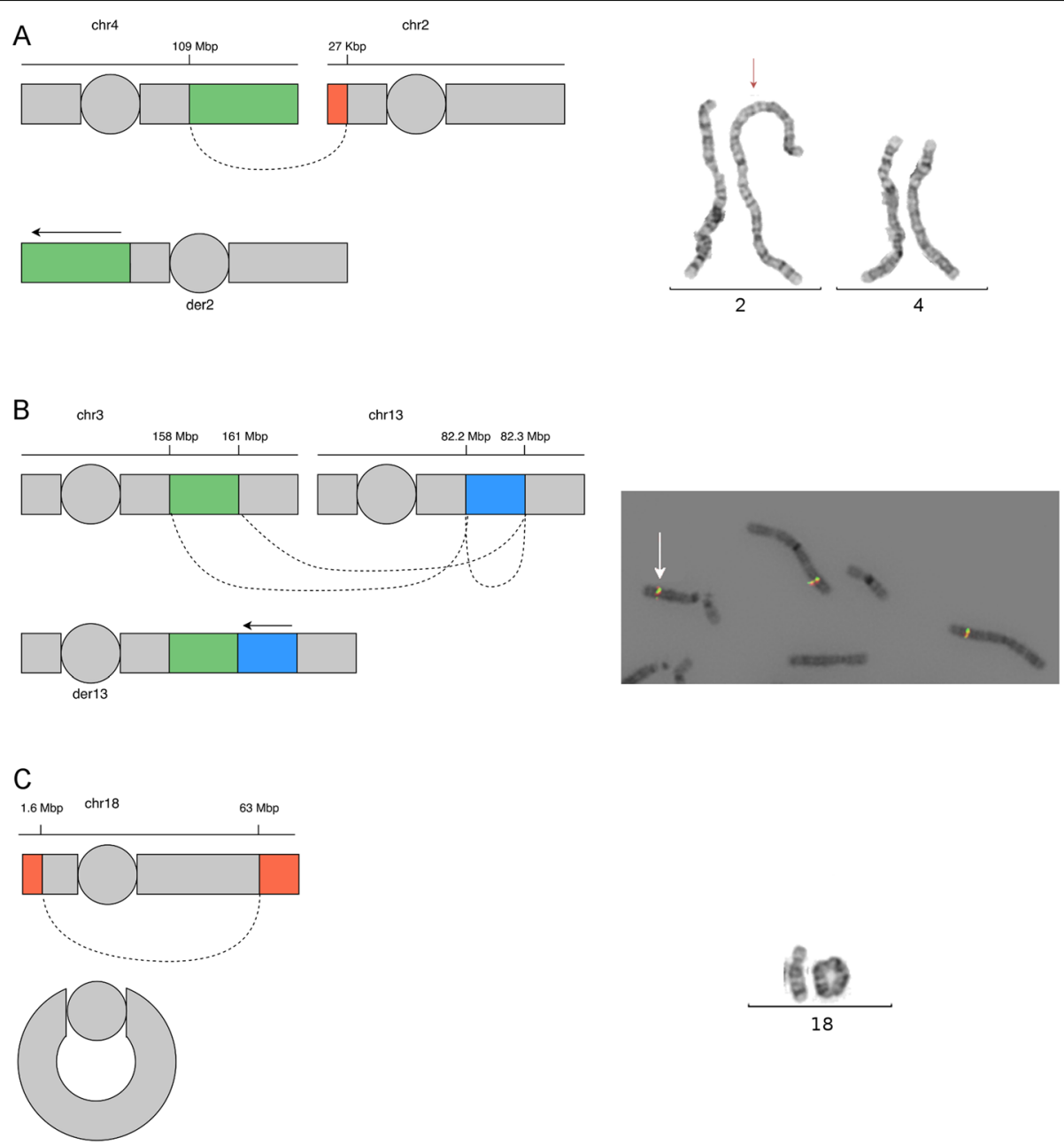

Fig. 3 Three cases with complex genomic rearrangements resolved by WGS. a A schematic drawing of the 4q25q35.2 unbalanced translocation in individual RD_P406. The duplicated segment of $81 \mathrm{~kb}$ (green) is inserted into the p-arm of chromosome 2 directly before the telomeric sequences. A 27-kb deletion on chromosome 2 (orange) is visible in the WGS data. The dashed line represents the links from chromosome 4 to chromosome 2. To the right, the insertional duplication rearrangement is shown through karyotyping with the derivative chromosome 2 indicated by a red arrow. $\mathbf{b}$ A schematic drawing of the 3q25.32q26.1 insertional duplication in individual RD_P405 as in $\mathbf{a}$. The duplicated segment of $2.23 \mathrm{Mb}$ is inserted into chromosome 13, and a genomic segment of $69.6 \mathrm{~kb}$ on chromosome 13, adjacent to the insertion, has been inverted. To the right, FISH analysis using probes RP11-209H21SG (green) and RP11-203L15SO (red) located within the rearranged region on chromosome 3. In addition to two signals from chr 3q25.32q26.1, an extra signal is present on chromosome 13 (white arrow) verifying the location of the duplicated segment. c A schematic drawing of the r(18) present in individual RD_P414 as in $\mathbf{a}$. To the right, the ring chromosome is shown through karyotyping

(VUS) $(n=2)$ (Table 4). Six rearrangements were recurrent known microdeletion syndromes: 7q11.23 deletions (RD_P407, RD_P408), 8p11.22 deletion (RD_P409), 15q13.2q13.3 deletion (RD_P412), 16p11.2 deletion (RD_P413), and 22q11.21 deletion (RD_P415) while the remainder were non-recurrent/private rearrangements. All of these CNVs were also detected by WGS. In addition, in individual RD_P417, the WGS-SV analysis detected a $3.7-\mathrm{kb}$ homozygous deletion of exon 2 in C12orf65 confirming a diagnosis of autosomal recessive spastic paraplegia 55 (SPG55) (MIM 615035) (Table 4). This deletion was not called by CMA due to insufficient probe coverage (no probes within the deleted regions).

\section{Comparison of WGS-SVs to CNVs detected by CMA}

After the array-CGH data was analyzed with standard clinical setting (three consecutive aberrant probes; $\log 2$ ratio cutoff -0.65 for deletions and 0.35 for duplications), a total of 2282 deletions and duplications were called in the 100 patients in cohort 3 (Additional file 3: Table S2). The median number of CNV calls was 22 (quantile (Q)1 18; Q3 26) with a median size of $51 \mathrm{~kb}$ (Q1 $24 \mathrm{~kb}$; Q3 $122 \mathrm{~kb}$ ). The corresponding numbers from WGS, after filtering for size $(>10 \mathrm{~kb}$ intergenic, and $>2 \mathrm{~kb}$ intergenic) and frequency $(<0.5 \%$ AF in SweFreq SVDB, $<1 \%$ in the internal SVDB), were a median number of 28 (Q1 20; Q3 36) and the median size of 17 

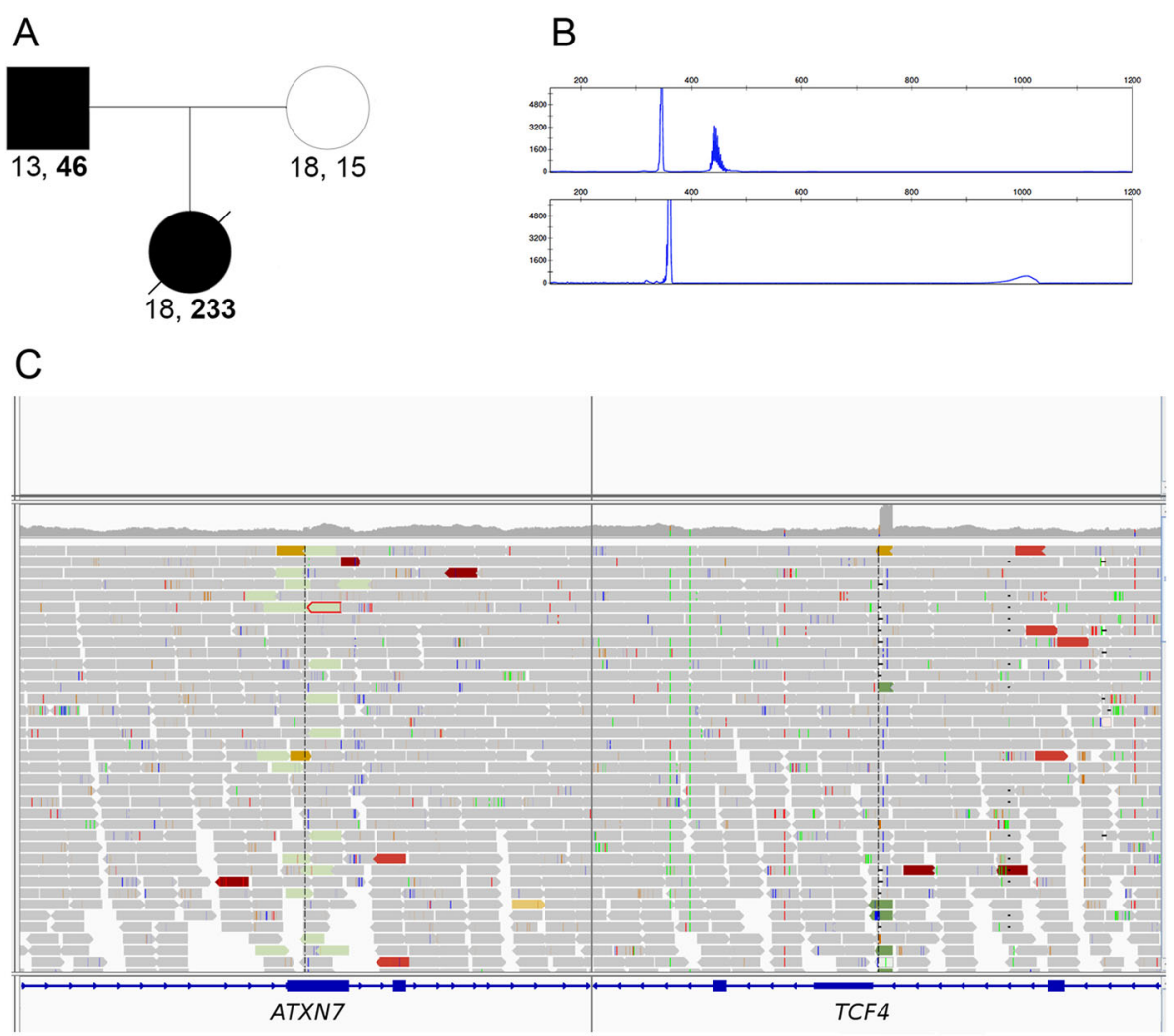

Fig. 4 A short tandem repeat expansion in ATXN7 is identified by WGS. a The pedigree and number of ATXN7 CAG repeats are illustrated under each individual. $\mathbf{b}$ The PCR-amplified CAG-repeat data from the father shows one normal sized allele and one expanded allele (top chromatogram). In the bottom chromatogram, the results from the affected child are shown. $\mathbf{c}$ Integrative Genomics Viewer (IGV) screenshot of the data obtained from FindSV shows the first indication of an ATXN7 abnormality. The aberrant signal was initially interpreted by the program as an insertion of sequence from chromosome 18 (right) into ATXN7 (left)

kb (Q1 $6 \mathrm{~kb}$; Q3 $70 \mathrm{~kb})$. Comparison of the output data files show that the filtered WGS-SVs only overlap with 9.1\% of the CMA calls (208/2282), and if we remove the frequency filter, the overlap increases $34.8 \%$ (794/2282) (Table S2). The CMA variants not detected by WGS were smaller, median $38 \mathrm{~kb}$ (Q1 19kb, Q3 98kb), compared to those also found with WGS, median $84 \mathrm{~kb}(\mathrm{Q} 1$ 41kb, Q3 154kb). In addition, the percentage of duplications among the CMA variants found by WGS was 52\% versus $75 \%$ among the variants not found by WGS.

\section{WGS reveals the presence of derivative chromosomes and solves their genomic structure}

In case RD_P414, the investigations with CMA identified two deletions on chromosome 18: $1.64 \mathrm{Mb}$ on the terminal p-arm and $15 \mathrm{Mb}$ on the terminal q-arm. Followup investigation with chromosome analysis showed that the rearrangement was in fact a ring chromosome present in $100 \%$ of the cells. In the same individual, blinded WGS analysis was able to detect the two deletions, characterize the rearrangement breakpoint junctions, and fully resolve the derivative chromosome structure (Fig. 3, Table 4, Additional file 2: Figure S4).

In two cases harboring duplications, the WGS analysis not only detected the presence of a duplication but also revealed that one duplication was part of a cryptic unbalanced translocation and the other was an insertional translocation. In individual RD_P406, an 81-Mb terminal duplication (4q25qter) had been inserted onto the p-arm of chromosome 2 directly before the telomeric sequences. WGS also uncovered a 27-kb deletion on 2p25.3pter not seen by CMA indicating that this was in fact an unbalanced translocation between chromosome 4q and chromosome 2p (Fig. 3, Table 4, Additional file 2: Figure S4). The breakpoint junction had a fournucleotide non-templated insertion in the junction, and sequence microhomology was low.

In individual RD_P405, a 2.23-Mb duplication of 3q25.32q26.1 was inserted into chromosome 13 (13q31.1). In addition, it was found that there were two breakpoints on chromosome 13, the genomic segment of $69.6 \mathrm{~kb}$ between them had been inverted, and the duplication originating from 3q25.32q26.1 was inserted into the proximal 


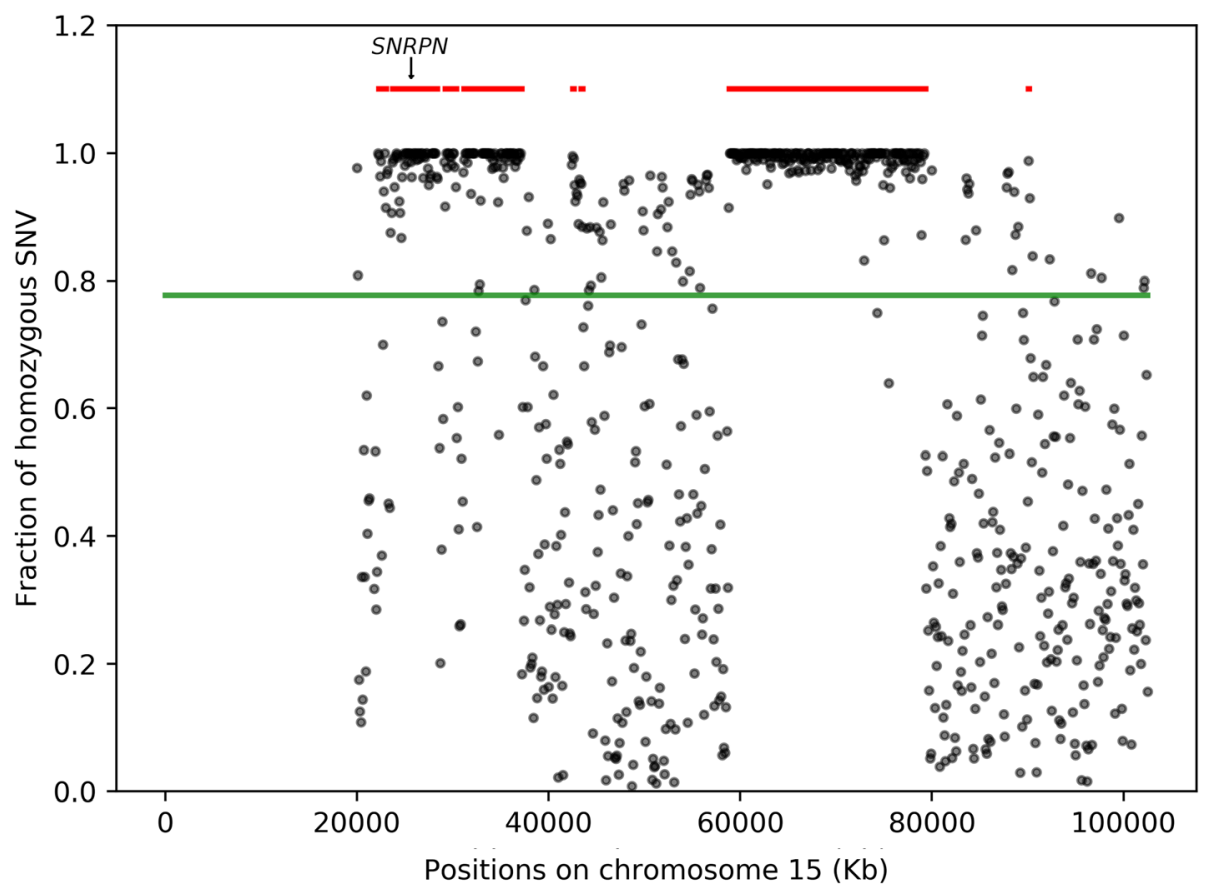

Fig. 5 Prader-Willi syndrome caused by maternal isodisomy. Homozygosity for SNPs on chromosome 15 from WGS data in individual RD_P432. The fraction of homozygous SNPs is shown on the $\mathrm{Y}$ axis and the position on chromosome 15 on the $\mathrm{X}$ axis. The position of SNRPN is indicated with an arrow. Each gray dot represents the fraction of homozygous SNVs in $10 \mathrm{~kb}$ regions. The green line indicates the fraction of homozygous SNV across the entire chromosome, and red lines indicate autozygous regions predicted by rhocall

breakpoint junction. This was only detected through WGS (Fig. 3). A total of three breakpoint junctions were identified in this patient, and the junctions showed little to no microhomology, no insertions, and no deletions (Table 4, Additional file 2: Figure S4).

Taken together, the breakpoint junctions from both patients harboring insertional duplications revealed no evidence for DNA replication errors, which has been the proposed mechanism underlying the formation of duplications in several cases $[68,78,79]$.

In summary, three out of 100 individuals (3\%) with intellectual disability referred for CMA carried derivative chromosomes with additional complexities detected and resolved by WGS.

\section{Single nucleotide variation}

The WGS data was next processed for SNVs and INDELs in a panel of 887 genes (Additional file 2: Document S1) as well as in custom panels created from HPO terms matching the individual patients' phenotypes. This way, heterozygous SNVs implicated in autosomal dominant disease were identified in six patients (6\%), of which five were classified as likely pathogenic and one as pathogenic. Compound heterozygous or homozygous SNVs implicated in autosomal recessive disease were identified in seven patients (7\%), of which one was classified as VUS, three as likely pathogenic and three as pathogenic. No X-linked variants were identified (Table 4).

\section{WGS diagnoses a lethal form of ataxia type 7 through a repeat expansion in ATXN7}

Next, we assessed the presence of STRs in 17 genes. A likely pathogenic repeat expansion in ATXN7 was identified in individual RD_P431 (Table 4). In brief, this was the second child born to unrelated parents. She was treated in the intensive care unit due to hypotonia, cardiac failure, and metabolic acidosis and died at the age of 10 months. The father had poor vision and balance problems but no molecular diagnosis. The detailed clinical description is available in the supplemental information (Additional file 2: Document S2). WGS was performed and filtered for genes involved in inborn errors of metabolism without identifying a causative genetic variant. Upon reanalysis of this case, no suspected pathogenic SNV, INDEL, or SV was identified. However, both FindSV (TIDDIT) and ExpansionHunter indicated an aberrant signal from the ATXN7 locus. The presence of a CAG STR was confirmed using PCR with fragment length analysis including triplet primed PCR. The detected number of 


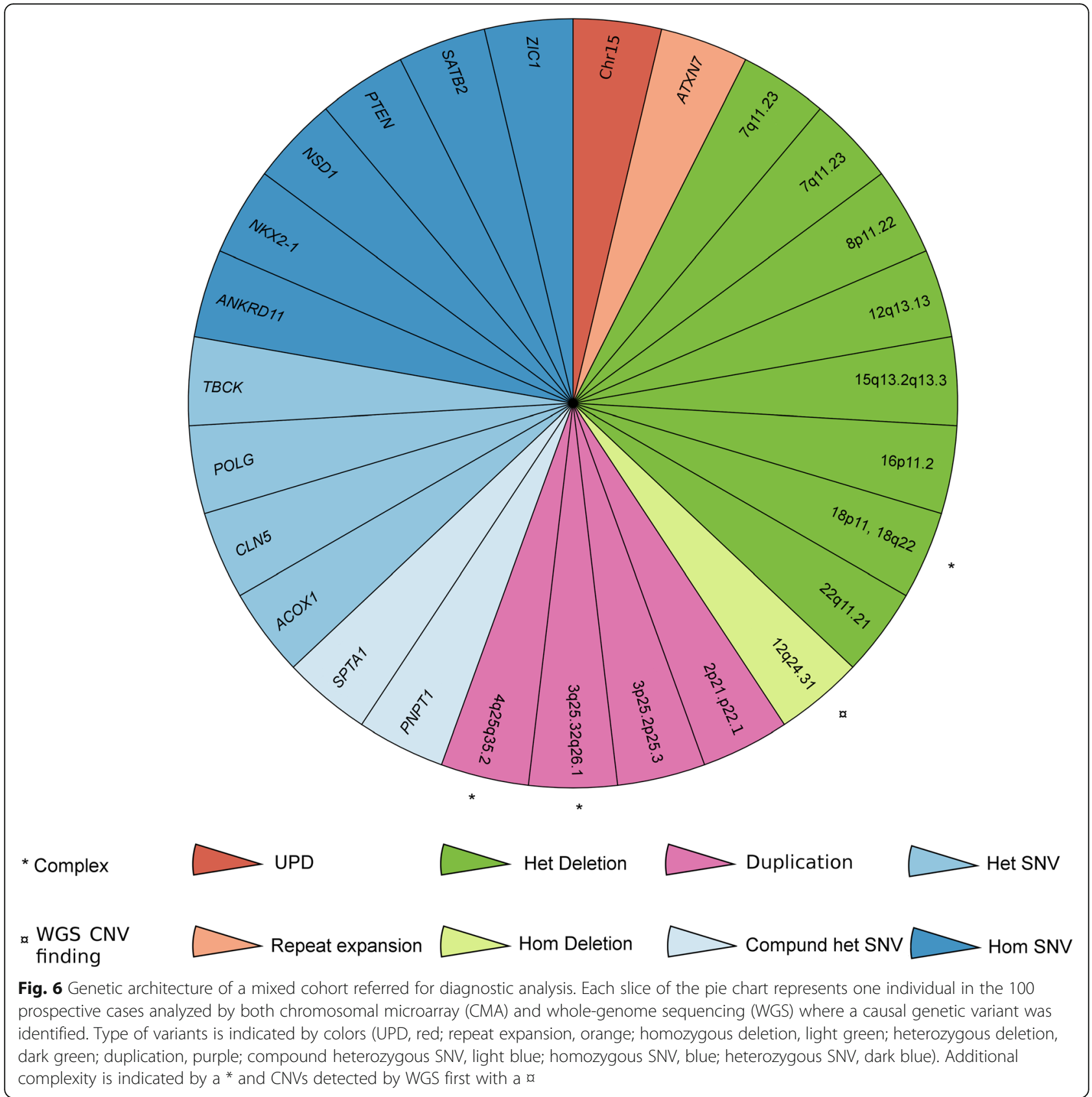

repeated CAG units was 233 in individual RD_P431 and 46 in the father confirming the diagnosis of Spinocerebellar ataxia 7 (SCA7) in both (MIM 164500) (Fig. 4).

\section{Maternal isodisomy is visible in the WGS data}

Genomic DNA from individual RD_P432 (from cohort 3) was analyzed with methylation-specific MLPA that showed a methylation pattern in the $15 \mathrm{q} 11.2 \mathrm{q} 13 \mathrm{re}$ gion consistent with a diagnosis of Prader-Willi syndrome (MIM 176270). Genotyping of 10 polymorphic markers located across chromosome 15 (from 22.9 to $49.3 \mathrm{Mb}$ ) in the patient and her mother suggested the presence of chromosome 15 maternal uniparental disomy (UPD) for the region 22.9-33.7 Mb. We were able to confirm this finding by assessing the B-allele frequency of SNPs on chromosome 15 in the WGS data. This analysis also confirmed that this was a case of segmental isodisomy (Fig. 5, Table 4).

Excluding the variants classified as VUS, the diagnostic yield in 100 unselected cases referred for CMA was 27\% compared to $12 \%$ with array only (Fig. 6). 


\section{Discussion}

Previous studies have suggested that short-read WGS could be a first-level test in individuals with neurodevelopmental delay and intellectual disability [80]. Here we show that WGS with regular coverage (30x) indeed captures the complex genetic variation underlying rare diseases in an unselected mixed cohort referred for genetic testing. Altogether, we found that $27 \%$ of these individuals harbored clinically relevant genetic variants that could be detected by WGS. In addition, the WGS analysis provided clinically important information by resolving the structure of the derivative chromosomes and revealing additional complexities.

Our data demonstrates that short-read WGS performs well for CNV detection from small intragenic variants to large chromosomal rearrangements. By applying multiple callers with complementary characteristics $[45,46]$, we successfully detected all 92 known SVs in our three cohorts as well as 11 previously undetected events. We previously compared the performance of the SV callers TIDDIT [46], Manta [81], CNVnator [45], DELLY [82], Fermikit [83], and Lumpy [84] and found that TIDDIT and CNVnator are complementary and offer high precision and sensitivity on large SV [46]. This was confirmed by a recent evaluation of the performance of $69 \mathrm{SV}$ detection algorithms including TIDDIT and CMVnator [85]. Therefore, we chose to combine the TIDDIT and CNVnator callers, enabling the detection of a wide range of variants while using a limited amount of computational resources. Overall, CNVnator [45] failed to detect five aberrations: three deletions (RD_P62, RD_P63, RD_ P64) and two duplications (RD_P123, RD_P139) all below $8 \mathrm{~kb}$ in size (Additional file 1: Table S1). This highlights that the coverage analysis has limitations in detecting such small CNVs. In contrast, TIDDIT [46] successfully identified all those small CNVs but were unable to call the recurrent events $(n=16)$. This is explained by the mechanism underlying the formation of those rearrangements, non-allelic homologous recombination between repeat structures flanking the CNV. Hence, the breakpoint junctions cluster in those repeats, and since TIDDIT uses split reads and discordant pairs to call an event, the read length $(151 \mathrm{bp})$ and insert size ( $350 \mathrm{bp}$ ) of short-read WGS are not enough to bridge the repetitive regions.

Of note, effective SV screening from WGS is highly dependent on the availability of high-quality frequency databases representing population background variation (and sequencing artifacts) which are essential for the selection of rare potentially disease-causing variants. We filtered the data against our internal database of $\sim 400$ individuals as well as the SV database from the 1000 Swedish genomes [6] that were analyzed with the same bioinformatic tools used here. After filtering for size (>
$10 \mathrm{~kb}$ genome wide and $>2 \mathrm{~kb}$ in target genes) and frequency (SweFreq SVDB $<0.5 \%$, internal SVDB $<1 \%$ ), we ended up with a manageable number of SV calls (median 38, average 54; standard deviation 48) that were moved forward to clinical interpretation. The standard deviation could be explained by some individuals of non-Swedish origin and sequencing quality.

The other main obstacle hampering the utility of WGS for SV screening in routine clinical diagnostics is visualization of the structural variants called by WGS. To overcome this, we developed vcf2cytosure [56], a program that converts VCF files with structural variations to the ".CGH" format. The output files are subsequently uploaded into the CytoSure Interpret Software, generally used for visualization of array-CGH data at our clinic (examples of output data visualized in this way are shown in the supplemental data; Additional file 2: Fig. S3). Through this approach, we facilitated the clinical interpretation of the WGS-SV data by non-bioinformaticians.

The 15 additional diagnoses provided by WGS compared to CMA included one homozygous exonic deletion, one STR, one UPD, and 12 SNVs (six heterozygous, four homozygous, and two compound heterozygous) (Table 4). In all cases, obtaining a diagnosis will have an immediate impact by providing more accurate information to the 15 families, enabling both carrier detection and prenatal testing. Embryo diagnostics will be possible for the nine couples with a high recurrence risk; seven with autosomal recessive cause of disease and two with autosomal dominant. In several cases, the molecular diagnosis leads to improved patient care and management, such as the father of RD_P431 with SCA7, and for others, the number of necessary hospital visits was decreased.

In aggregate, our data show that WGS has the potential to be a single test for the detection of the many different genetic variation types underlying rare diseases. However, for each variant class (SVs, SNVs, STRs, $\mathrm{ROH}$, and mosaic events), it is important to understand the limitations of the test. For the calling of SNVs and INDELs, WGS has already shown high sensitivity and specificity [86], but both SVs, STR, and $\mathrm{ROH}$ analysis need to be further studied in larger cohorts. Hence, due to a lack of data on sensitivity and specificity of WGS for calling SVs, STRs, and $\mathrm{ROH}$, it is important to remember that a normal result is still hard to interpret and we suggest that all such cases are clinically assessed and if necessary, offered additional testing.

Our evaluation here of WGS-SV analysis in a clinical setting has provided several important insights. First, the detection of 107 disease-causing CNVs (Table 2, Table 3, Table 4, Additional file 1: Table S1) illustrates that WGS can be used to detect disease-causing CNVs of different sizes and types. Second, comparing the WGS-SVs in 
Table 5 Complex rearrangements detected in the current study

\begin{tabular}{|c|c|c|c|c|}
\hline Case & Cohort & Type & Chromosome(s) & Phenotype \\
\hline RD_P22 & Cohort 1 & DEL-NML-DEL & 5 & NDD \\
\hline RD_P54 & Cohort 1 & DEL-INV-DEL & 17 & NDD \\
\hline RD_P07 & Cohort 1 & DEL-NML-DEL-NML-DUP & 1 & $\mathrm{NDD}+$ \\
\hline RD_P05 & Cohort 1 & DEL-DUP-DEL & 2 & Internal malformations \\
\hline RD_P26 & Cohort 1 & DEL-INV-DEL & 21 & NDD+ \\
\hline RD_P105 & Cohort 1 & DUP-NML-DUP & 7 & NDD \\
\hline RD_P106 & Cohort 1 & DUP-NML-DUP & 14 & NDD \\
\hline RD_P77 & Cohort 1 & DEL-T & 4,7 & NDD+ \\
\hline RD_P393 & Cohort 2 & DEL-INV-DEL & 2 & Epilepsy \\
\hline RD_P405 & Cohort 3 & DUP-INS & 3,13 & Growth retardation \\
\hline RD_P406 & Cohort 3 & DUP-INS & 4,2 & NDD, microcephaly \\
\hline RD_P414 & Cohort 3 & Ring chromosome & 18 & NDD \\
\hline
\end{tabular}

DEL deletion, NML normal, INV inversion, $T$ translocation, INS insertion, NDD neurodevelopemental disorder, NDD+ syndromic NDD

cohort 3 to all the CNVs called by CMA in the same individuals, we find that the two methods not only produce different amounts of variants but also that only one third of the polymorphic CNVs called by CMA are present in the WGS files (Additional file 3: Table S2). This is problematic, however, we cannot be entirely sure as to which calls are true, since the WGS data is compared to array-CGH data which is derived from relative quantification. Furthermore, the CMA calls not detected by WGS are smaller (median $38 \mathrm{~kb}$ vs $84 \mathrm{~kb}$ for CNVs detected by WGS) and prone towards duplications ( $75 \%$ vs $52 \%$ in CNVs detected by WGS) supporting the notion that these are indeed technical artifacts in the CMA data.

The data presented here also illustrate that screening for STRs and ROH using WGS is a feasible and fruitful approach increasing the number of patients with a definite molecular diagnosis. We consider it clinically relevant to perform these analyses in patients with unexplained intellectual disability. Clinical labs already perform targeted testing for the FMR1 repeat expansion (FRAX, MIM 309548) and UPD 15 (Angelman syndrome, MIM 105830; Prader-Willi syndrome, MIM 176270) in such cases. If this information is obtainable in the WGS data, it should also be analyzed.

The mosaic trisomy 9 in individual RD_P167 show similar levels of mosaicism by CMA and WGS, and the discrepancy with FISH is likely a culture artifact. The sensitivity of WGS to detect mosaicism still needs to be further evaluated and will most likely depend on the coverage, the caller(s) used, and the specific chromosome affected. Both size and quality of the reference genome as well as GC content will most likely influence the performance. In a previous publication, we assessed the detection rate of a simulated trisomy $\mathrm{X}$ and show that it can be detected confidently down to $10 \%$ with $30 \times$ WGS [67].

The diagnostic yield obtained here (27\%) using WGS as a first-tier diagnostic test should be compared to previous studies obtaining over 30\% diagnostic yield after SNV analysis of WES data [17]. Since WGS is free from the capture biases that may affect WES and offers the possibility to detect more types of variants (i.e., balanced chromosomal rearrangements, small CNVs affecting single exons, repeat expansions, and deep splice variants), one might expect a higher diagnostic yield. However, the diagnostic yield of WES may range from 8 [87] to 60\% [88], depending on the selection criteria and whether proband-only or familial WES was performed [15]. Here we studied a randomly selected cohort of 100 individuals referred for CMA to our center and we only sequenced the probands. After this first screening analysis, the negative cases with a high probability of a genetic cause may be offered trio WGS analysis. In this way, we expect the diagnostic yield to rise, mainly due to an increased detection rate of de novo missense mutations in known disease-causing genes but also due to a higher power to detect variation in new undescribed diseasecausing genes as well as in non-exonic regions of established genes.

As has been shown previously [29, 78, 89, 90], our data confirms the notion that structural variants are important contributors also to Mendelian diseases (12/156, 7.7\%). The LAMA2 duplication identified in RD_P394 and RD_P395 may represent a founder mutation. As we assess structural variation in more individuals, both healthy and clinically affected, the true frequency of rare founder SVs will also be revealed. This is important and needs to be taken into 
consideration as we transition to whole-genome diagnostic sequencing.

Several cases in the three studied cohorts harbored known or unexpected complex structural variants. The high accuracy of WGS allowed us to map the breakpoint junctions with nucleotide resolution and study mutational signatures. In the validation cohort, in addition to eight cases of complex intrachromosomal rearrangements that were reported previously [68], a $9.3-\mathrm{Mb}$ de novo deletion on chromosome 4 detected in individual RD_P77 was in fact part of a reciprocal translocation between chromosomes 4 and 7 (Fig. 1; Additional file 1: Table S1, Additional file 2: Document S2). The breakpoint junction analysis revealed no microhomology and non-templated insertions of random nucleotides, indicative of NHEJ repair of double-strand breaks. The complex 2q24.3 rearrangement involving three Mendelian epilepsy genes identified in individual RD_P393 (Fig. 2, Table 3) showed templated insertions in both breakpoint junctions, causing two small segments of $11 \mathrm{bp}$ and 13 bp each to be duplicated. The mutational signatures suggest a replicative error as the mechanism of formation, such as fork stalling and template switching (FoSTeS) [91]. Finally, in the 100 individuals included in the prospective cohort, one ring chromosome (RD_P414) one unbalanced translocation (RD_P406), and one insertional translocation (RD_P405) were resolved (Fig. 3). Each one of the three cases is of a distinct type, and no conclusive underlying mechanism was highlighted from the breakpoint junction analysis.

The ability to outline structural rearrangement connectivity pictures also adds support for the use of WGS as a first-line test in intellectual disability. We have shown previously that this information is important for a proper interpretation of intragenic duplications [78] as well as complex genomic rearrangements (CGRs) [68]. CGRs were a common finding in all three cohorts $(8 / 68 ; 1 / 156 ; 3 / 100)$ highlighting that in cases with a genetic disease caused by an SV, the probability of detecting a complex rearrangement is substantial; 12/96 SVs were detected in all three cohorts (12.5\%) (Table 2, Table 3, Table 4, Table 5). In a cohort of 100 patients with intellectual disabilities not previously studied by any technique, WGS detected derivative chromosomes accompanied by additional complexities in 3\%. Even in individuals with clinical symptoms not expected to be caused by CNVs (such as the cases in cohort 2), the probability is not negligible $(0.6 \%)$. This type of information would not be provided by most other technologies including CMA.

De novo CGRs, which may be seen as two or more de novo CNVs in the genome, can be observed in $\sim 2 \%$ of patients with clinical indication to undergo array studies [92] and are more common in some loci associated with genomic disorders. In $M E C P 2$ duplication syndrome (MIM 300260) at Xq28 [93, 94] and Pelizaeus-Merzbacher disease (MIM 312080) due to increased PLP1 copy number at Xq22 [94-96], specific CGRs account for up to 30\% of the pathological SVs. In autosomal loci, those CGRs represent a lower number of pathological SVs $(<20 \%)$ [97, 98], although technical ascertainment may explain lower detection. Even apparently "simple" non-recurrent rearrangements may actually consist of complex breakpoint junctions formed by multiple insertions of short templated segments (<100 nucleotides), which was shown in 27 to $35 \%$ of simple CNV junctions in disease-associated loci as well as polymorphic CNVs [99]. Moreover, inversions, which cannot be detected by arrays, are associated with CGRs in 84 [100] to $100 \%$ of the cases [94, 95, 99].

\section{Conclusions}

In conclusion, our data show that WGS robustly not only captures SNVs but also performs well for the detection of disease-causing CNVs and has the potential to detect STRs, $\mathrm{ROH}$, and chromosomal rearrangements. These findings demonstrate that WGS may be used as a single test instead of performing two separate analyses to detect SVs and SNVs, such as CMA followed by WES, in addition to targeted analyses for specific repeat expansions and UPDs. Even though further studies are necessary to fully understand the limitations of WGS and how to interpret a normal result, for clinics already using clinical WGS for SNV analysis, the added value of mining the data for additional types of disease-causing mutations is high.

\section{Supplementary information}

Supplementary information accompanies this paper at https://doi.org/10. 1186/s13073-019-0675-1.

Additional file 1: Table S1. Detailed information of individuals in Cohort 1.

Additional file 2: Figure S1. Flowchart showing different filtering steps of WGS SV analysis, Figure S2. Flowchart showing different filtering steps of WGS SNV analysis. Document S1. List of genes in the intellectual disability panel. Figure S3. Vcf2cytosure and array plots of illustrative cases. Figure S4. Breakpoint junction analysis of individuals RD_P77, RD_P393, RD_P400 and RD_P431. Document S2. Detailed clinical descriptions of individuals RD_P77, RD_P393, RD_P400 and RD_P431.

Additional file 3: Table S2. Number of detected CNVs using array and WGS.

\section{Abbreviations}

WGS: Whole-genome sequencing; SNV: Single nucleotide variant; CNV: Copy number variant; SV: Structural variant; HPO: Human Phenotype Ontology; STR: Short tandem repeat; INDEL: Small insertions and deletions;

MPS: Massively parallel sequencing; CMA: Chromosomal microarray analysis; FISH: Fluorescent in situ hybridization; WES: Whole-exome sequencing;

UPD: Uniparental disomy; array-CGH: Array comparative genomic hybridization; MLPA: Multiplex ligation-dependent probe amplification; ACMG: American College of Medical Genetics and Genomics; MAF: Minor allele frequency; ExAC: Exome Aggregation Consortium; RoH: Run of homozygozity; NHEJ: Non-homologous end-joining; VUS: Variants of 
uncertain significance; CGR: Complex genomic rearrangement; F: Female; M: Male; NDD: Neurodevelopmental disorder; CTD: Connective tissue disorder; SKD: Skeletal dysplasia; NMD: Neuromuscular disease

\section{Acknowledgements}

We are very grateful to the participating families. We would also like to thank UPPMAX for the use of computer infrastructure resources and the support from the National Genomics Infrastructure (NGI) Stockholm and Clinical Genomics Stockholm facility at the Science for Life Laboratory in providing assistance in massive parallel sequencing.

\section{Authors' contributions}

AL conceptualized the study, analyzed and interpreted data, and wrote the manuscript. JE and MP analyzed and interpreted data and contributed to the writing of the manuscript. JE, MåM, MaM, HS, and DN performed bioinformatics development. BMA, OB, CMBC, GG, PG, AH, PGH, El, MJS, MK, $\mathrm{KL}, \mathrm{AgL}, \mathrm{HM}, \mathrm{MN}, \mathrm{AnN}, \mathrm{AmN}, \mathrm{ES}, \mathrm{ET}$, JW, SY, and AW provided patient samples, clinical information of patients, and/or analysis and interpretation of data. DN, JL, and WW conceptualized the study, analyzed and interpreted the data, and were major contributors in writing of the manuscript. All authors have read, edited, and approved the final manuscript.

\section{Funding}

AL was supported by grants from the SciLifeLab national sequencing grant and the Stockholm County Council. AL and AN were supported by grants from the Swedish Research Council and the Swedish Brain Foundation. AN was supported by grants from The Hållsten Research Foundation. MP was supported by grants from Karolinska Institutet funding for doctoral education (KID) and The Royal Physiographic Society in Lund (Nilsson-Ehle donations). MaM was supported by the Knut and Alice Wallenberg Foundation as part of the National Bioinformatics Infrastructure Sweden at SciLifeLab.

\section{Availability of data and materials}

The datasets supporting the conclusions of this article are included within the article and its additional files. All variants reported have been submitted to ClinVar [62], accession number SCV000897707 (ATXN7 expansion) and submission number SUB5433665. The consent provided by the research subjects did not permit sharing of the entire genome-wide data set. The inhouse databases used in this article also contain information from clinical samples and are not publicly available due to compromise of patient confidentiality.

The following public databases and open source software were used: Genome Reference Consortium Human Build 37 (https://www.ncbi.nIm.nih. gov/assembly/GCF_000001405.13/) [36].

The Swedish variant frequency database (SweFreq) [60] and the Swedish structural variant frequency database (SweFreq SVDB) [49], both available from https://swefreq.nbis.se/ [6].

The Human Phenotype Ontology (HPO) term database (http://compbio. charite.de/hpoweb/) [55].

The Genomics England panel app (https://panelapp.genomicsengland.co. uk/) [52].

The ClinVar database (https://www.ncbi.nlm.nih.gov/clinvar/) [62].

The Online Mendelian Inheritance in Man (OMIM; https://www.omim.org)

[40].

The University of California Santa Cruz (UCSC) Genome Browser (www. genome.ucsc.edu) [65].

The Database of Genomic Variants (DGV; http://dgv.tcag.ca) [38].

The Database of Chromosomal Imbalance and Phenotype in Humans using Ensembl Resources (DECIPHER; http://decipher.sanger.ac.uk) [39].

Exome Aggregation Consortium (ExAC v0.2; http://exac.broadinstitute.org/) [59]

The Genome Aggregation Database (gnomAD; https://gnomad. broadinstitute.org/) [70].

FindSV pipeline (https://github.com/J35P312/FindSV) [44].

SVDB (https://github.com/J35P312/SVDB) [47].

FreeBayes (https://arxiv.org/abs/1207.3907) [51].

vcf2cytosure (https://github.com/NBISweden/vcf2cytosure) [56].

rhocall (https://github.com/dnil/rhocall) [63].

\section{Ethics approval and consent to participate}

The Regional Ethical Review Board in Stockholm, Sweden, approved the study (ethics permit numbers KS 2012/222-31/3 and 2012/2106-31/4). This ethics permit allows for use of clinical samples for analysis of scientific importance as part of clinical development. Included subjects were part of clinical cohorts investigated at our center. IRB approval for informed consent to participate in the study was waived; written informed consent was obtained for those participants for whom potentially identifiable clinical information was included in the manuscript. The research conformed to the principles of the Helsinki Declaration.

\section{Consent for publication}

Written informed consent was obtained to publish clinical information.

\section{Competing interests}

The authors declare that they have no competing interests.

\section{Author details}

Department of Clinical Genetics, Karolinska University Hospital, Stockholm, Sweden. ${ }^{2}$ Department of Molecular Medicine and Surgery, Karolinska Institutet, Stockholm, Sweden. ${ }^{3}$ Center for Molecular Medicine, Karolinska Institutet, Stockholm, Sweden. ${ }^{4}$ Science for Life Laboratory, Karolinska Institutet, Stockholm, Sweden. ${ }^{5}$ Department of Molecular and Human Genetics, Baylor College of Medicine, Houston, Texas, USA. ${ }^{6}$ The Department of Women's and Children's Health, Karolinska Institutet, Stockholm, Sweden. ${ }^{7}$ Centre for Inherited Metabolic Diseases, Karolinska University Hospital, Stockholm, Sweden. ${ }^{8}$ Department of Biochemistry and Biophysics, National Bioinformatics Infrastructure Sweden, Science for Life Laboratory, Stockholm University, Stockholm, Sweden. ${ }^{9}$ Science for Life Laboratory, School of Engineering Sciences in Chemistry, Biotechnology and Health, KTH Royal Institute of Technology, Stockholm, Sweden. ${ }^{10}$ Science for Life Laboratory, Department of Microbiology, Tumor and Cell biology, Karolinska Institutet, Stockholm, Sweden.

Received: 7 May 2019 Accepted: 9 October 2019

Published online: 07 November 2019

\section{References}

1. Boycott KM, Rath A, Chong JX, Hartley T, Alkuraya FS, Baynam G, et al. International cooperation to enable the diagnosis of all rare genetic diseases. Am J Hum Genet. 2017;100(5):695-705.

2. Genomes Project C, Abecasis GR, Auton A, Brooks LD, DePristo MA, Durbin RM, et al. An integrated map of genetic variation from 1,092 human genomes. Nature. 2012;491(7422):56-65.

3. Mills RE, Walter K, Stewart C, Handsaker RE, Chen K, Alkan C, et al. Mapping copy number variation by population-scale genome sequencing. Nature. 2011;470(7332):59-65.

4. Feuk L, Carson AR, Scherer SW. Structural variation in the human genome. Nat Rev Genet. 2006; 7(2):85-97.

5. Maretty L, Jensen JM, Petersen B, Sibbesen JA, Liu S, Villesen $P$, et al. Sequencing and de novo assembly of 150 genomes from Denmark as a population reference. Nature. 2017:548(7665):87-91.

6. Ameur A, Dahlberg J, Olason P, Vezzi F, Karlsson R, Martin M, et al. SweGen: a whole-genome data resource of genetic variability in a cross-section of the Swedish population. Eur J Hum Genet. 2017;25(11):1253-60.

7. Genomes Project C, Auton A, Brooks LD, Durbin RM, Garrison EP, Kang HM, et al. A global reference for human genetic variation. Nature. 2015; 526(7571):68-74.

8. Lek M, Karczewski KJ, Minikel EV, Samocha KE, Banks E, Fennell T, et al. Analysis of protein-coding genetic variation in 60,706 humans. Nature. 2016; 536(7616):285-91.

9. Audano PA, Sulovari A, Graves-Lindsay TA, Cantsilieris S, Sorensen M, Welch $A E$, et al. Characterizing the major structural variant alleles of the human genome. Cell. 2019;176(3):663-75 e19.

10. Beaudet AL. The utility of chromosomal microarray analysis in developmental and behavioral pediatrics. Child Dev. 2013;84(1):121-32.

11. Miller DT, Adam MP, Aradhya S, Biesecker LG, Brothman AR, Carter NP, et al. Consensus statement: chromosomal microarray is a first-tier clinical diagnostic test for individuals with developmental disabilities or congenital anomalies. Am J Hum Genet. 2010;86(5):749-64.

12. Waggoner D, Wain KE, Dubuc AM, Conlin L, Hickey SE, Lamb AN, et al. Yield of additional genetic testing after chromosomal microarray for diagnosis of neurodevelopmental disability and congenital anomalies: a clinical practice 
resource of the American College of Medical Genetics and Genomics (ACMG). Genet Med. 2018;20(10):1105-13.

13. Hartman P, Beckman K, Silverstein K, Yohe S, Schomaker M, Henzler C, et al. Next generation sequencing for clinical diagnostics: five year experience of an academic laboratory. Mol Genet Metab Rep. 2019;19:100464.

14. Krumm N, Sudmant PH, Ko A, O'Roak BJ, Malig M, Coe BP, et al. Copy number variation detection and genotyping from exome sequence data. Genome Res. 2012;22(8):1525-32.

15. Retterer K, Juusola J, Cho MT, Vitazka P, Millan F, Gibellini F, et al. Clinical application of whole-exome sequencing across clinical indications. Genet Med. 2016;18(7):696-704

16. Rossi M, El-Khechen D, Black MH, Farwell Hagman KD, Tang S, Powis Z. Outcomes of diagnostic exome sequencing in patients with diagnosed or suspected autism spectrum disorders. Pediatr Neurol. 2017:70:34-43 e2.

17. Srivastava S, Love-Nichols JA, Dies KA, Ledbetter DH, Martin CL, Chung WK, et al. Meta-analysis and multidisciplinary consensus statement: exome sequencing is a first-tier clinical diagnostic test for individuals with neurodevelopmental disorders. Genet Med. 2019.

18. Dharmadhikari AV, Ghosh R, Yuan B, Liu P, Dai H, Al Masri S, et al. Copy number variant and runs of homozygosity detection by microarrays enabled more precise molecular diagnoses in 11,020 clinical exome cases. Genome Med. 2019;11(1):30.

19. Bowling KM, Thompson ML, Amaral MD, Finnila CR, Hiatt SM, Engel KL, et al. Genomic diagnosis for children with intellectual disability and/or developmental delay. Genome Med. 2017;9(1):43.

20. Gu S, Posey JE, Yuan B, Carvalho CM, Luk HM, Erikson K, et al. Mechanisms for the generation of two quadruplications associated with split-hand malformation. Hum Mutat. 2016;37(2):160-4.

21. Eldomery MK, Coban-Akdemir Z, Harel T, Rosenfeld JA, Gambin T, StrayPedersen $A$, et al. Lessons learned from additional research analyses of unsolved clinical exome cases. Genome Med. 2017:9(1):26.

22. Jehee FS, de Oliveira VT, Gurgel-Giannetti J, Pietra RX, Rubatino FVM, Carobin NV, et al. Dual molecular diagnosis contributes to atypical Prader-Willi phenotype in monozygotic twins. Am J Med Genet A. 2017;173(9):2451-5.

23. Karaca E, Posey JE, Coban Akdemir Z, Pehlivan D, Harel T, Jhangiani SN, et al. Phenotypic expansion illuminates multilocus pathogenic variation. Genet Med. 2018;20(12):1528-37.

24. Gross AM, Ajay SS, Rajan V, Brown C, Bluske K, Burns NJ, et al. Copy-number variants in clinical genome sequencing: deployment and interpretation for rare and undiagnosed disease. Genet Med. 2019;21(5):1121-30.

25. Trost B, Walker S, Wang Z, Thiruvahindrapuram B, MacDonald JR, Sung WWL, et al. A comprehensive workflow for read depth-based identification of copy-number variation from whole-genome sequence data. Am J Hum Genet. 2018;102(1):142-55.

26. Ellingford JM, Campbell C, Barton S, Bhaskar S, Gupta S, Taylor RL, et al. Validation of copy number variation analysis for next-generation sequencing diagnostics. Eur J Hum Genet. 2017;25(6):719-24.

27. Nilsson D, Pettersson M, Gustavsson P, Forster A, Hofmeister W, Wincent J, et al. Whole-genome sequencing of cytogenetically balanced chromosome translocations identifies potentially pathological gene disruptions and highlights the importance of microhomology in the mechanism of formation. Hum Mutat. 2017:38(2):180-92.

28. Bramswig NC, Ludecke HJ, Pettersson M, Albrecht B, Bernier RA, Cremer K, et al. Identification of new TRIP12 variants and detailed clinical evaluation of individuals with non-syndromic intellectual disability with or without autism. Hum Genet. 2017;136(2):179-92.

29. Grigelioniene G, Nevalainen Pl, Reyes M, Thiele S, Tafaj O, Molinaro A, et al. A large inversion involving GNAS exon A/B and all exons encoding Gsalpha is associated with autosomal dominant pseudohypoparathyroidism type $\mathrm{lb}$ (PHP1B). J Bone Miner Res. 2017;32(4):776-83.

30. Dolzhenko E, van Vugt J, Shaw RJ, Bekritsky MA, van Blitterswijk M, Narzisi G, et al. Detection of long repeat expansions from PCR-free whole-genome sequence data. Genome Res. 2017:27(11):1895-903.

31. Scocchia A, Wigby KM, Masser-Frye D, Del Campo M, Galarreta Cl, Thorpe $E_{\text {, et }}$ al. Clinical whole genome sequencing as a first-tier test at a resource-limited dysmorphology clinic in Mexico. NPJ Genom Med. 2019:4:5.

32. Gilissen C, Hehir-Kwa JY, Thung DT, van de Vorst M, van Bon BW, Willemsen $\mathrm{MH}$, et al. Genome sequencing identifies major causes of severe intellectual disability. Nature. 2014;511(7509):344-7.
33. Tsai EA, Shakbatyan R, Evans J, Rossetti P, Graham C, Sharma H, et al. Bioinformatics Workflow for Clinical Whole Genome Sequencing at Partners HealthCare Personalized Medicine. J Pers Med. 2016;6(1):12.

34. Stranneheim $H$, Engvall $M$, Naess $K$, Lesko $N$, Larsson $P$, Dahlberg $M$, et al. Rapid pulsed whole genome sequencing for comprehensive acute diagnostics of inborn errors of metabolism. BMC Genomics. 2014;15:1090.

35. Noll AC, Miller NA, Smith LD, Yoo B, Fiedler S, Cooley LD, et al. Clinical detection of deletion structural variants in whole-genome sequences. NPJ Genom Med. 2016;1:16026.

36. Genome Reference Consortium Human Build 37. https://www.ncbi.nlm.nih. gov/assembly/GCF_000001405.13/. Accessed 21 Oct 2019.

37. Kearney HM, Thorland EC, Brown KK, Quintero-Rivera F, South ST. Working Group of the American College of Medical Genetics Laboratory Quality Assurance C. American College of Medical Genetics standards and guidelines for interpretation and reporting of postnatal constitutional copy number variants. Genet Med. 2011;13(7):680-5.

38. The Database of Genomic Variants. http://dgv.tcag.ca. Accessed 21 Oct 2019.

39. The Database of Chromosomal Imbalance and Phenotype in Humans using Ensembl Resources. http://decipher.sanger.ac.uk. Accessed 21 Oct 2019.

40. The Online Mendelian Inheritance in Man. https://www.omim.org. Accessed 21 Oct 2019.

41. National Genomics Infrastructure (NGI), Stockholm, Sweden. https:// ngisweden.scilifelab.se/. Accessed 21 Oct 2019

42. Clinical Genomics, Stockholm, Sweden. https://www.scilifelab.se/facilities/ clinical-genomics-stockholm/. Accessed 21 Oct 2019.

43. DePristo MA, Banks E, Poplin R, Garimella KV, Maguire JR, Hartl C, et al. A framework for variation discovery and genotyping using next-generation DNA sequencing data. Nat Genet. 2011;43(5):491-8.

44. The FindSV pipeline. https://github.com/J35P312/FindSV. Accessed 17 Dec 2018

45. Abyzov A, Urban AE, Snyder M, Gerstein M. CNVnator: an approach to discover, genotype, and characterize typical and atypical CNVs from family and population genome sequencing. Genome Res. 2011;21(6):974-84.

46. Eisfeldt J, Vezzi F, Olason P, Nilsson D, Lindstrand A. TIDDIT, an efficient and comprehensive structural variant caller for massive parallel sequencing data. F1000Res. 2017:6:664.

47. SVDB. https://github.com/J35P312/SVDB. Accessed 20 Sept 2018.

48. McLaren W, Gil L, Hunt SE, Riat HS, Ritchie GR, Thormann A, et al. The Ensembl variant effect predictor. Genome Biol. 2016;17(1):122.

49. The Swedish structural variant frequency database. https://swefreq.nbis.se/. Accessed 21 Oct 2019

50. Li H, Handsaker B, Wysoker A, Fennell T, Ruan J, Homer N, et al. The sequence alignment/map format and SAMtools. Bioinformatics. 2009;25(16):2078-9.

51. FreeBayes. https://arxiv.org/abs/1207.3907. Accessed 21 Oct 2019.

52. Genomics England panel app. https://panelapp.genomicsengland.co.uk/. Accessed 1 Jan 2018.

53. Kohler S, Carmody L, Vasilevsky N, Jacobsen JOB, Danis D, Gourdine JP, et al. Expansion of the Human Phenotype Ontology (HPO) knowledge base and resources. Nucleic Acids Res. 2019:47(D1):D1018-D27.

54. Kohler S, Doelken SC, Mungall CJ, Bauer S, Firth HV, Bailleul-Forestier I, et al. The Human Phenotype Ontology project: linking molecular biology and disease through phenotype data. Nucleic Acids Res. 2014;42(Database issue):D966-74

55. Human Phenotype Ontology database. http://compbio.charite.de/hpoweb/. Accessed 1 Jan 2018.

56. vcf2cytosure. https://github.com/NBISweden/vcf2cytosure. Accessed 21 Oct 2019

57. Richards S, Aziz N, Bale S, Bick D, Das S, Gastier-Foster J, et al. Standards and guidelines for the interpretation of sequence variants: a joint consensus recommendation of the American College of Medical Genetics and Genomics and the Association for Molecular Pathology. Genet Med. 2015; 17(5):405-24.

58. Eisfeldt J, Pettersson M, Vezzi F, Wincent J, Kaller M, Gruselius J, et al. Comprehensive structural variation genome map of individuals carrying complex chromosomal rearrangements. PLoS Genet. 2019;15(2):e1007858.

59. Exome Aggregation Consortium (ExAC v0.2), http://exac.broadinstitute.org/. Accessed 21 Oct 2019

60. The Swedish variant frequency database. https://swefreq.nbis.se/. Accessed 21 Oct 2019.

61. Internal SNV scoring system. Ihttps:/github.com/Clinical-Genomics/MIP/blob/ master/templates/rank_model_cmms_-v1.25-ini. Accessed 21 Oct 2019. 
62. The ClinVar database. https://www.ncbi.nlm.nih.gov/clinvar/. Accessed 21 Oct 2019

63. rhocall. https://github.com/dnil/rhocall. Accessed 1 Jan 2018.

64. Kent WJ. BLAT--the BLAST-like alignment tool. Genome Res. 2002;12(4):656-64.

65. The UCSC Genome Browser. http://www.genome.ucsc.edu/. Accessed 21 Oct 2019.

66. Sequeiros J, Seneca S, Martindale J. Consensus and controversies in best practices for molecular genetic testing of spinocerebellar ataxias. Eur J Hum Genet. 2010;18(11):1188-95.

67. Eisfeldt J, Nilsson D, Andersson-Assarsson JC, Lindstrand A. AMYCNE: confident copy number assessment using whole genome sequencing data. PLoS One. 2018;13(3):e0189710

68. Nazaryan-Petersen L, Eisfeldt J, Pettersson M, Lundin J, Nilsson D, Wincent J, et al. Replicative and non-replicative mechanisms in the formation of clustered CNVs are indicated by whole genome characterization. PLoS Genet. 2018;14(11):e1007780.

69. Chiang C, Jacobsen JC, Ernst C, Hanscom C, Heilbut A, Blumenthal I, et al. Complex reorganization and predominant non-homologous repair following chromosomal breakage in karyotypically balanced germline rearrangements and transgenic integration. Nat Genet. 2012;44(4):390-7 S1.

70. The Genome Aggregation Database (gnomAD). https://gnomad. broadinstitute.org/. Accessed 21 Oct 2019.

71. Helbling-Leclerc A, Zhang X, Topaloglu H, Cruaud C, Tesson F, Weissenbach J, et al. Mutations in the laminin alpha 2-chain gene (LAMA2) cause merosindeficient congenital muscular dystrophy. Nat Genet. 1995;11(2):216-8.

72. Hisado-Oliva A, Ruzafa-Martin A, Sentchordi L, Funari MFA, Bezanilla-Lopez C, Alonso-Bernaldez M, et al. Mutations in C-natriuretic peptide (NPPC): a novel cause of autosomal dominant short stature. Genet Med. 2018;20(1):91-7.

73. Krejci $P$, Masri B, Fontaine V, Mekikian PB, Weis $M$, Prats $H$, et al. Interaction of fibroblast growth factor and $\mathrm{C}$-natriuretic peptide signaling in regulation of chondrocyte proliferation and extracellular matrix homeostasis. J Cell Sci. 2005:118(Pt 21):5089-100.

74. Bartnik M, Chun-Hui Tsai A, Xia Z, Cheung SW, Stankiewicz P. Disruption of the SCN2A and SCN3A genes in a patient with mental retardation, neurobehavioral and psychiatric abnormalities, and a history of infantile seizures. Clin Genet. 2011;80(2):191-5.

75. Fry AE, Rees E, Thompson R, Mantripragada K, Blake P, Jones G, et al. Pathogenic copy number variants and SCN1A mutations in patients with intellectual disability and childhood-onset epilepsy. BMC Med Genet. 2016;17(1):34.

76. Harkin LA, McMahon JM, lona X, Dibbens L, Pelekanos JT, Zuberi SM, et al. The spectrum of SCN1A-related infantile epileptic encephalopathies. Brain. 2007;130(Pt 3):843-52

77. Vanoye CG, Gurnett CA, Holland KD, George AL Jr, Kearney JA. Novel SCN3A variants associated with focal epilepsy in children. Neurobiol Dis. 2014;62:313-22.

78. Pettersson M, Vaz R, Hammarsjo A, Eisfeldt J, Carvalho CMB, Hofmeister W, et al. Alu-Alu mediated intragenic duplications in IFT81 and MATN3 are associated with skeletal dysplasias. Hum Mutat. 2018;39(10):1456-67.

79. Gu S, Szafranski P, Akdemir ZC, Yuan B, Cooper ML, Magrina MA, et al. Mechanisms for complex chromosomal insertions. PLoS Genet. 2016;12(11): e1006446.

80. Clark MM, Stark Z, Farnaes L, Tan TY, White SM, Dimmock D, et al. Metaanalysis of the diagnostic and clinical utility of genome and exome sequencing and chromosomal microarray in children with suspected genetic diseases. NPJ Genom Med. 2018;3:16.

81. Chen X, Schulz-Trieglaff O, Shaw R, Barnes B, Schlesinger F, Kallberg M, et al. Manta: rapid detection of structural variants and indels for germline and cancer sequencing applications. Bioinformatics. 2016;32(8):1220-2.

82. Rausch T, Zichner T, Schlattl A, Stutz AM, Benes V, Korbel JO. DELLY: structural variant discovery by integrated paired-end and split-read analysis. Bioinformatics. 2012;28(18):i333-i9.

83. Li H. FermiKit: assembly-based variant calling for Illumina resequencing data. Bioinformatics. 2015;31(22):3694-6.

84. Layer RM, Chiang C, Quinlan AR, Hall IM. LUMPY: a probabilistic framework for structural variant discovery. Genome Biol. 2014;15(6):R84.

85. Kosugi S, Momozawa Y, Liu X, Terao C, Kubo M, Kamatani Y. Comprehensive evaluation of structural variation detection algorithms for whole genome sequencing. Genome Biol. 2019;20(1):117.

86. Eberle MA, Fritzilas E, Krusche P, Kallberg M, Moore BL, Bekritsky MA, et al. A reference data set of 5.4 million phased human variants validated by genetic inheritance from sequencing a three-generation 17-member pedigree. Genome Res. 2017;27(1):157-64.
87. Tammimies K, Marshall CR, Walker S, Kaur G, Thiruvahindrapuram B, Lionel $A C$, et al. Molecular diagnostic yield of chromosomal microarray analysis and whole-exome sequencing in children with autism spectrum disorder. JAMA. 2015;314(9):895-903.

88. Anazi S, Maddirevula S, Faqeih E, Alsedairy H, Alzahrani F, Shamseldin $H E$, et al. Clinical genomics expands the morbid genome of intellectual disability and offers a high diagnostic yield. Mol Psychiatry. 2017;22(4):615-24.

89. Lindstrand A, Frangakis S, Carvalho CM, Richardson EB, McFadden KA, Willer $J R$, et al. Copy-number variation contributes to the mutational load of Bardet-Biedl syndrome. Am J Hum Genet. 2016;99(2):318-36.

90. Lieden A, Kvarnung M, Nilssson D, Sahlin E, Lundberg ES. Intragenic duplication--a novel causative mechanism for SATB2-associated syndrome. Am J Med Genet A. 2014;164A(12):3083-7.

91. Lee JA, Carvalho CM, Lupski JR. A DNA replication mechanism for generating nonrecurrent rearrangements associated with genomic disorders. Cell. 2007;131(7):1235-47.

92. Liu P, Yuan B, Carvalho CMB, Wuster A, Walter K, Zhang L, et al. An organismal CNV Mutator phenotype restricted to early human development. Cell. 2017;168(5):830-42 e7.

93. Carvalho CM, Pehlivan D, Ramocki MB, Fang P, Alleva B, Franco LM, et al. Replicative mechanisms for CNV formation are error prone. Nat Genet. 2013; 45(11):1319-26.

94. Carvalho CM, Ramocki MB, Pehlivan D, Franco LM, Gonzaga-Jauregui C, Fang $P$, et al. Inverted genomic segments and complex triplication rearrangements are mediated by inverted repeats in the human genome. Nat Genet. 2011:43(11):1074-81.

95. Beck CR, Carvalho CM, Banser L, Gambin T, Stubbolo D, Yuan B, et al. Complex genomic rearrangements at the PLP1 locus include triplication and quadruplication. PLoS Genet. 2015;11(3):e1005050.

96. Carvalho CM, Bartnik M, Pehlivan D, Fang P, Shen J, Lupski JR. Evidence for disease penetrance relating to CNV size: Pelizaeus-Merzbacher disease and manifesting carriers with a familial $11 \mathrm{Mb}$ duplication at Xq22. Clin Genet. 2012;81(6):532-41.

97. Gu S, Yuan B, Campbell IM, Beck CR, Carvalho CM, Nagamani SC, et al. Alumediated diverse and complex pathogenic copy-number variants within human chromosome 17 at p13.3. Hum Mol Genet. 2015;24(14):4061-77.

98. Yuan B, Harel T, Gu S, Liu P, Burglen L, Chantot-Bastaraud S, et al. Nonrecurrent 17p11.2p12 rearrangement events that result in two concomitant genomic disorders: the PMP22-RAl1 contiguous gene duplication syndrome. Am J Hum Genet. 2015;97(5):691-707.

99. Carvalho CM, Lupski JR. Mechanisms underlying structural variant formation in genomic disorders. Nat Rev Genet. 2016;17(4):224-38.

100. Collins RL, Brand H, Redin CE, Hanscom C, Antolik C, Stone MR, et al. Defining the diverse spectrum of inversions, complex structural variation, and chromothripsis in the morbid human genome. Genome Biol. 2017; 18(1):36.

\section{Publisher's Note}

Springer Nature remains neutral with regard to jurisdictional claims in published maps and institutional affiliations.

Ready to submit your research? Choose BMC and benefit from:

- fast, convenient online submission

- thorough peer review by experienced researchers in your field

- rapid publication on acceptance

- support for research data, including large and complex data types

- gold Open Access which fosters wider collaboration and increased citations

- maximum visibility for your research: over $100 \mathrm{M}$ website views per year

At $\mathrm{BMC}$, research is always in progress.

Learn more biomedcentral.com/submission 